\title{
Subdifferentials of Compactly Lipschitzian Vector-Valued Functions (*).
}

\author{
Lroner Thibault (Pau, France)
}

\begin{abstract}
Summary. - We introduce the concept of compactly lipschitzian functions taling values in a topological vector space $F$. We show that if $F$ is finite dimensional the Lipschitz functions are compactly lipschitizian. We define the notions of generalized directional derivatives and subdifferentials for such functions $f$ taking values in an ordered topological vector space. It is shown that this notion of subdifferential coincides with the one of $F$. H. Clarke when $f$ is Lispchitz and $F=\mathbb{R}$. Some formulas for this subdifferential concerning the cases of finite sum, composition, pointwise supremum and continuous sum are studied.
\end{abstract}

\section{Introduction.}

The theory of generalized gradients of real-valued locally Lipschitz functions built up first by F. H. Clarke has been the subject of much development; for the results, we refer to CLARKE [6] for the finite dimensional case and AUBTN [1], ClaRKe [7], Lebourg [20] and Thibautr [40] for the infinite dimensional case. Here, our aim is to develop a theory of subdifferentials for a class of vector valued functions extending the one of real-valued locally Lipschitz functions.

In the last few years, in order to obtain optimality conditions for infinitely, many inequality constraints many authors have investigated vector-valued functions and defined for them a notion of directional derivative. The first ones to have considered such functions seem to be RAFFIN [34], VALADIER [43], IOFFE and LeVIN [15] and Zows [46] who have extended the concepts of directional derivative and subdifferential of a convex function. In [30] J. P. PENot has introduced the notions of upper and lower directional derivatives of a vector-valued function and considered the functions for which these directional derivatives are sublinear. In [3], for a point $\bar{x}$ in a topological vector space $E$, M. S. BAzARAA and J. J. Goode have used vectorvalued functions $f$ for which the mappings

$$
v \mapsto \lim _{\substack{t \downarrow 0 \\ c \rightarrow v}} t^{-1}[f(\bar{x}+t n)-f(\bar{x})]
$$

have a sense and are sublinear. P. MICHEL has investigated in [24] vector-valued functions $f$ such that $f=g+d$ where $g$ is convex and $d$ is strictly differentiable.

In this paper, after recent works, for instance, these of AUSLENDER [2], CLARkE [6-9], Goldstein [10], HiRiart-UrRuty [12-14], Lebourg [20], Muffiti [25],

(*)' Entrata in Redazione il 24 aprile 1979. 
Pourdiau [33], Thibault [40 42], which have shown that the generalized gradient of Clarke has many important applications, we introduce a class of vector-valued functions which contains the class of Lipschitz functions when the range space is the real line. For these functions we define a notion of subdifferential which coincides with the one of generalized gradient of F. H. CLARKE $[6,7]$ for a real-valued locally Lipschitz function.

The first section deals with the definitions of compactly lipschitzian mappings and strictly lipschitzian mappings. It is shown that the set of mappings of a topological vector space into another one which are compactly lipschitzian at a fixed point is a vector space. Properties of composition are studied and some examples are given. It is also proved that under some conditions a convex vectorvalued function is compactly lipschitzian.

In $\S 2$ we define the directional derivative of a compactly lipschitzian mapping which takes values in an ordered topological vector space. Some important properties of this directional derivative are studied, in particular it is shown that it is a sublinear mapping.

The third section is concerned with the definition of the subdifferential of a compactly lipschitzian mapping defined on a vector space $E$. Conditions under which the subdifferential at a point is a non empty convex compact subset for the pointwise convergence of the space of continuous linear mappings of $E$ into the range vector space are given. It is also proved that the convex subdifferential of a convex compactly lipschitzian mapping coincides with the above subdifferential.

Some formulas for this subdifferential concerning the cases of finite sum, composition and pointwise supremum are studied in $\S 4$. The results that we obtain are generalizations of known results for real-valued Lipschitz functions. We also apply the result about the composition with a positive continuous linear functional to the study of a continuous sum of real-valued Lipschitz functions.

$\S 5$ is devoted to the study of the subdifferential of a continuous sum of compactly lipschitzian mappings.

Before concluding this introduction, let us indicate that all topological vector spaces that we shall consider will be Hausdorff real topological vector spaces and that we shall always assume that the positive cone of an ordered topological vector space is closed.

\section{1. - Compactly Lipschitzian mappings.}

Throughout the following $E$ and $F$ will be two topological vector spaces and $\mathbb{R}$ will denote the real number system.

\subsection{Defrnitions.}

1) Let $f$ be a mapping of $E$ into $F$ and let $\bar{x}$ be a point in $E$. We shall say that $f$ is compactly lipsehitzian at the point $\bar{x}$ if there are a mapping $K$ of $E$ into the 
set Comp $(F)$ of nonempty compact subsets of $F$ and a mapping $r$ of $] 0,1] \times E \times E$ into $F$ which verify the following properties:

a) $\lim r(t, x ; v)=0$ for each $v \in E$; $\underset{x \rightarrow \bar{x}}{t \downarrow 0}$

b) for each $v \in E$ there exist a neighborhood $\Omega$ of $\bar{x}$ in $E$ and a real number $\eta \in] 0,1]$ such that

$t^{-1}[f(x+t v)-f(x)] \in K(v)+r(t, x ; v) \quad$ for all $x \in \Omega$ and $\left.\left.t \in\right] 0, \eta\right]$.

2) $f$ will be locally compactly lipschitzian on $E$, if it is compactly lipschitzian at every point $x \in E$.

Let us also note that for a lot of mappings $f$ that we shall encounter in the sequel, there are mappings $K$ and $r$ and neighborhoods $\Omega$ of $\bar{x}$ and $V$ of zero in $E$ satisfying:

$\left.a^{\prime}\right) \quad \lim r(t, x ; v)=0$ for each $v \in V$ and $\lim r(t, x ; v)=0$; $\underset{\substack{t \downarrow 0 \\ x \rightarrow \bar{x}}}{\substack{t \downarrow 0 \\ x \rightarrow \bar{x} \\ y \rightarrow 0}}$

$\left.b^{\prime}\right)$ for all $x \in \Omega, v \in V$ and $\left.\left.t \in\right] 0,1\right]$

$$
t^{-1}[f(x+t v)-f(x)] \in K(v)+r(t, x ; v)
$$

$\left.e^{\prime}\right) \cdot K(0)=\{0\}$ and the multifunction $K$ is upper semi-continuous at the origin (that is for each neighborhood $W$ of $K(0)$ in $F$ there is a neighborhood $U$ of zero in $E$ verifying $K(v) \subset W$ for every $v \in U$ ).

We shall say that these mappings are strictly lipschitzian at the point $\bar{x}$.

REMarK. - It is not difficult to see that a mapping which is strictly lipschitzian at a point is compactly lipschitzian at this point.

Before proceeding to the definition of the subdifferential of a compactly lipschitzian mapping, we shall give some properties and some examples.

The first one will be that a mapping which is strictly lipschitzian at a point $\bar{x}$ is Lipschitz at $\bar{x}$ in a certain sense.

In order to deal with let us recall the following definition.

If $U$ is a closed, circled neighborhood of the origin in a topological vector space $X$. the gauge of $U$ is the real-valued function, $\varrho_{\sigma}$ defined by

$$
\varrho_{U}(x)=\inf \{t>0 \mid x \in t U\} \quad \text { for every } x \in X \text {. }
$$

It is easy to see that the function $\varrho_{U}$ is positively homogeneous and continuous at the origin with $\varrho_{U}(0)=0$ and that $x \in U$ if and only if $\varrho_{U}(x) \leqslant 1$. 
1.2. Proposttron. - If $f$ is a mapping of $E$ into $F$ which is strictly lipschitzian at $\vec{x}$, then $f$ is Lipschitz at $\vec{x}$ in the sense that for every closed, circled neighborhood $W$ of the origin in $F$ there are a closed, circled neighborhood $U$ of the origin in $E$ and $a$ neighborhood $X$ of $\vec{x}$ in $E$ such that

$$
\varrho_{W}(f(y)-f(x)) \leqslant \varrho_{V}(y-x) \quad \text { for all } x, y \in X
$$

Proof. - Let $W$ be any closed, circled neighborhood of the origin in $F$. Consider a neighborhood $\Omega$ of $\vec{x}$ and a neighborhood $V$ of zero in $E$ defined by condition $\left(a^{\prime}\right)$ in definition 1.1 and choose a neighborhood $W_{1}$ of zero in $F$ verifying $W_{1}+W_{1} \subset W$. The definition of a strictly lipschitzian mapping implies that there exist a circled neighborhood $U$ of zero in $E$ and a positive real number $\gamma<1$ such that

$$
U \subset V, \quad \bar{x}+U \subset \Omega
$$

and

$$
K(v) \subset W_{1} \quad \text { and } \quad r(t, x ; v) \in W_{1}
$$

for all $t \in] 0, \gamma], x \in \ddot{x}+U$ and $v \in U$. Then, by condition $\left(b^{\prime}\right)$ we have

$$
f(x+w)-f(x) \in t W
$$

for all $x \in \bar{x}+V, w \in t U$ and $t \in] 0, \gamma]$. Choose a neighborhood $X$ of $\bar{x}$ satisfying $X-X \subset 2^{-1} \gamma U$. If $x$ and $y$ are two points in $X$ and if $\lambda$ is any real number verifying

$$
\varrho_{v}(y-x)<\lambda \leqslant 2^{-1} \gamma
$$

then we have

$$
f(y)-f(x)=f(x+(y-x))-f(x) \in \lambda W
$$

for $y-x \in \lambda U$ and $\lambda<\gamma$. So we have

$$
\varrho_{w}(f(y)-f(x)) \leqslant \lambda
$$

and hence

$$
\varrho_{W}(f(y)-f(x)) \leqslant \varrho_{v}(y-x)
$$

REMARK. - If $E$ and $F$ are two normed vector spaces it is an easy matter to verify that the definition of Lipschitz mappings given above in terms of gauges is equivalent to the usual definition.

As a consequence of the above proposition we have the following property. 
1.3. Corollary. - If a mapping $f$ of $E$ into $F$ is strictly lipschitzian at $\vec{x}$, then it is continuous at $\vec{x}$.

REMARK. - A mapping $f$ which is compactly lipschitzian at a point $\bar{x}$ is not necessarily continuous at this point. For instance, let $E$ be the space of all sequences of real numbers $\left(x_{n}\right)_{n \in \mathbb{N}}$ with $x_{n} \neq 0$ only for a finite number of integers $n$ endowed with the norm $\|x\|=\max _{n \in \mathbb{N}}\left|x_{n}\right|$. Define a real-valued function $f$ on $E$ by putting

$$
f(x)=x_{0}^{2}+\sum_{n \geqslant 0} n x_{n} \quad \text { for } x=\left(x_{n}\right)_{n \in \mathbb{N}}
$$

Then $f$ is compactly lipschitzian and non-continuous at the origin (and hence non strictly lipschitzian at this point).

1.4. Proposition. - Assume that $F$ is a dual Banach space, that is the topological dual of a Banach space. If a mapping $f$ of $E$ into $F$ is Lipschitz at $\bar{x}$ with respect to the strong topology of $F$, then $f$ is strictly lipschitzian at $\bar{x}$ with respect to the weak star topology of $\boldsymbol{F}$.

Proof. - If $f$ is Lipschitz at $\bar{x}$, there are a closed circled neighborhood $U$ of the origin, a neighborhood $X$ of $\bar{x}$ in $E$ verifying

$$
\|f(y)-f(x)\| \leqslant \varrho_{U}(y-x) \quad \text { for all } y, x \text { in } X
$$

Choose a neighborhood $\Omega$ of $\bar{x}$ and a circled neighborhood $V$ of zero in $E$ verifying $\Omega+V \subset X$ and take

$$
r \equiv 0 \quad \text { and } K(v)=\varrho_{v}(v) \bar{B}(0,1)
$$

where $\bar{B}(0,1)=\{z \in F \mid\|z\| \leqslant 1\}$. Since $\bar{B}(0,1)$ is weakly star compact in $F$ and that $\varrho_{U}$ is positively homogeneous and continuous at the origin, the mappings $K$ and $r$ and the neighborhoods $\Omega$ and $V$ satisfy the conditions of the definition of a strictly lipschitzian mapping.

1.5. Corollary. - If $F$ is a finite dimensional vector space, then a mapping $f$ of $E$ into $F$ is strictly lipschitzian at a point if and only if it is Lipschitz at this point.

Proof. - It is a simple consequence of propositions 1.2 and 1.4 .

1.6. Proposition. - Assume that $f$ and $g$ are two mappings of $E$ into $F$ compactly lipschitzian at $\vec{x} \in E$, that $B$ (resp. A) is a continuous affine mapping of a topological 
vector space $H$ into $E$ (resp. a continuous linear mapping of $F$ into a topological vector space $G$ ) and that $B \bar{y}=\bar{x}$, then the following assertions hold:

i) $f+g$ is compactly lipschitzian at the point $\bar{x}$;

ii) $A \circ f \circ B$ is compactly lipschitzian at the point $\bar{y}$.

Proof. - Consider a mapping $K_{f}\left(\right.$ resp. $K_{g}$ ) of $E$ into Comp $\left(F^{\prime}\right)$ and a mapping $r_{f}$ (resp. $r_{g}$ ) of $\left.] 0,1\right] \times E \times E$ into $F$ verifying the above conditions for the mapping $f$ (resp. $g$ ) and the point $\bar{x}$. It is easily seen that the mappings $K_{f}+K_{g}$ and $r_{f}+r_{g}$ satisfy conditions $(a)$ and $(b)$ of definition 1.1 for the mapping $f+g$ and the point $\bar{x}$. Now, let $v$ be a point in $H$. Write $B=b+\bar{B}$ where $b \in E$ and $\bar{B}$ is a linear mapping of $H$ into $E$. There exist a neighborhood $\Omega$ of $\vec{x}$ and a real number $\eta \in] 0,1]$ such that

$$
\left.\left.t^{-1}[f(x+t \bar{B} v)-f(x)] \in K_{f}(\bar{B} v)+r_{f}(t, x ; \bar{B} v) \quad \text { for each }(t, x) \in\right] 0, \eta\right] \times \Omega .
$$

If we choose a neighborhood $W$ of $\bar{y}$ with $B(W) \subset \Omega$ and if we put $K(\approx)=$ $=A\left(K_{f} \circ \bar{B}(z)\right)$ for each $z \in E$ and $r(t, y ; v)=A\left(r_{f}(t, B y ; \bar{B} v)\right)$, then we have

$$
\left.\left.t^{-1}[A \circ f \circ B(y+t v)-A \circ f \circ B(y)] \in K(v)+r(t, y ; v) \quad \text { for each }(t, y) \in\right] 0, \eta\right] \times W,
$$

and the proposition is proved.

REVIARK. - Similar results also hold in the strictly lipschitzian case.

Let us give some other examples of compactly lipschitzian mappings.

\subsection{Examples.}

a) If a mapping $f$ of a normed vector space $E$ into a topological vector space $F$ is strictly differentiable at a point $\bar{x} \in E$, that is if there is a continuous linear mapping $\nabla f(\bar{x})$ of $E$ into $F^{\prime}$ such that

$$
\lim _{\substack{x \rightarrow \bar{x} \\ y \rightarrow \bar{x} \\ x \neq y}}[f(x)-f(y)-\nabla f(\bar{x}) \cdot(x-y)] /\|x-y\|=0 \quad \text { (see [4]), }
$$

then $f$ is strictly lipschitzian at $\bar{x}$. Indeed, if we put $K(v)=\{\nabla f(\bar{x}) \cdot v\}$ and $r(t, x ; v)=$ $=t^{-1}[f(x+t v)-f(x)-t \nabla f(\vec{x}) \cdot v]$ it is an easy matter to verify that the mappings $K$ and $r$ satisfy the conditions of the definition of a strictly lipsehitzian mapping.

b) Suppose that $F$ is an ordered topological vector space in which the order intervals $\left[z_{1}, z_{2}\right]=\left\{z \in F \mid z_{1} \leqslant z \leqslant z_{2}\right\}$ are compact. Let $h$ be a mapping of $E$ into $F$ which is positively homogeneous, that is, $h(t v)=t h(v)$ for all $t \geqslant 0$ and $v \in E$. If $f$ is a mapping of $E$ into $F$ and if there exists a neighborhood $\Omega$ of a point $\vec{x} \in E$ such that $f(x)-f(y) \leqslant h(x-y)$ for all $x \in \Omega$ and $y \in \Omega$, then $f$ is compactly lipschitzian 
at $\bar{x}$. Indeed, it is easily seen that $f(x)-f(y) \in[-h(y-x), h(x-y)]$ for all $x \in \Omega$ and $y \in \Omega$. If we put $K(v)=[-h(-v), h(v)]$, the elements $K$ and $r \equiv 0$ satisfy the conditions of definition 1.1 for the mapping $f$ and the point $\bar{x}$.

c) Let $G, E$ be two normed vector spaces and let $F$ be an ordered topological vector space. Consider a mapping $g$ of $G$ into $E$ which is Lipsehitz at a point $\bar{x}$, a mapping $f$ of $E$ into $F$ such that there exist a neighborhood $W$ of $g(\vec{x})$ and a point $k$ in the positive cone $F_{+}=\{z \in F \mid z \geqslant 0\}$ of $F$ which satisfy the following relation:

$$
f\left(y_{1}\right)-f\left(y_{2}\right) \leqslant\left\|y_{1}-y_{2}\right\| k \text { for all } y_{1} \in W \text { and } y_{2} \in W \text {. }
$$

If we suppose that the order intervals of $F$ are compact, then the mapping $f \circ g$ is compactly lipschitzian at the point $\bar{x}$. Indeed, since $g$ is Lipschitz at $\bar{x}$, there exist a real number $\alpha>0$ and a neighborhood $\Omega$ of $\vec{x}$ such that $g(\Omega) \subset W$ and $\left\|g\left(x_{1}\right)-g\left(x_{2}\right)\right\| \leqslant \alpha\left\|x_{1}-x_{2}\right\|$ for all $x_{1} \in \Omega$ and $x_{2} \in \Omega$. Therefore, it follows that

$$
f \circ g\left(x_{1}\right)-f \circ g\left(x_{2}\right) \leqslant\left\|g\left(x_{1}\right)-g\left(x_{2}\right)\right\| k \leqslant \alpha\left\|x_{1}-x_{2}\right\| k
$$

for all $x_{1} \in \Omega$ and $x_{2} \in \Omega$. Define a mapping $h$ of $G$ into $F$ by $h(v)=\alpha\|v\| k$ for each $v \in G$. Then we see that the mapping $h$ and the neighborhood $\Omega$ of $\vec{x}$ satisfy the conditions of example $(b)$ for the mapping $f \circ g$ and the point $\bar{x}$.

If, in addition, the positive cone $F_{+}$is normal (see 1.8), then $f \circ g$ is strictly lipsehitzian at $\bar{x}$.

Before making some remarks, we recall the following definitions.

1.8. Definttions. - The positive cone $F_{+}$of an ordered topological vector space $F$ is normal if there exists a neighborhood basis $\{V\}_{F}$ of the origin in $F$ such that

$$
V=\left(V+F_{+}\right) \cap\left(V-F_{+}\right) \text {. }
$$

We say that an ordered topological vector space $F$ is normal if the positive cone $F_{+}$is normal.

Let us note that if $F_{+}$is normal and if $\left(x_{j}\right)_{j \in J},\left(y_{j}\right)_{j \in J},\left(z_{j}\right)_{j \in J}$ are three nets in $F^{\prime}$ verifying $x_{j} \leqslant y_{j} \leqslant z_{j}$ for all $j \in J$ and $\lim _{j \in J} x_{j}=\lim _{j \in J} z_{j}=0$, then we have $\lim _{j \in J} y_{j}=0$ (see [32]).

\section{REMARIS.}

1) Let $F$ be a strongly closed subspace of the dual of a Banach space. Suppose that $F$ is equipped with the topology induced by the weak star topology and that $F$ is ordered by a strongly closed convex cone $F_{+}$. If the positive cone $F_{+}$is normal for the norm topology of $F$, then the order intervals $\left[z_{1}, z_{2}\right]$ are bounded in $F$ (see $[32]$ ), hence compact in $F$ for the weak star topology. For instance, if $\mu$ is a positive measure on a tribe $\Sigma$ of a set $S$, the space $L^{p}(S, \Sigma, \mu)$ of all equivalence classes of real- 
valued $\mu$-measurable functions $f$ such that $|f|^{p}$ is $\mu$-integrable if $1<p<+\infty$ or $\mu$-essentially bounded if $p=+\infty$ verify these assumptions for its natural positive cone.

2) If $F=L_{\mathbf{R}}^{1}(S, \Sigma, \mu)$, the order intervals of $F$ are weakly compact in $F$ since they are equi-integrable subsets of $L_{\mathbf{R}}^{1}(S, \Sigma, \mu)$.

3) If $p$ is a positive integer and $F=l^{p}$, the space of all sequences $\left(x_{n}\right)_{n \in \mathbb{N}}$ such that $\sum_{n \in \mathbb{N}}\left|x_{n}\right|^{p}<\infty$ endowed with the norm $\|x\|_{j}=\left[\sum_{n \in \mathbb{N}}\left|x_{n}\right|^{p}\right]^{1 / p}$ and its natural order, it is an easy matter to verify that the order intervals are compact.

Now we are going to study the case where $f$ is a convex vector-valued function which is continuous on a neighborhood of $\bar{x}$ in $E$.

Let us recall that for a topological vector lattice (see [32]) the lattice operations

$$
x \mapsto \sup (x, 0) \quad \text { and } \quad x \mapsto \text { in }(x, 0)
$$

are continuous and the positive cone is normal.

1.9. Proposition. - Assume that $F$ is an ordered topological vector lattice for which the order intervals are $\theta$-compact, where $\theta$ is a vector topology on $F$ which is coarser than the topology of $F$. If $f$ is a mapping of $E$ into $F$ and if $\bar{x}$ is a point in $E$ for which there exists an open convex set $U$ with $\vec{x} \in U$ and such that the restriction of $f$ on $U$ is convex and continuous, then $f$ is strictly lipschitzian at $\bar{x}$ with respect to the $\theta$-topolology.

Proof. - Choose a neighborhood $Q$ of $\bar{x}$ and a circled neighborhood $V$ of zero in $E$ verifying $\Omega+V \subset U$. Since the restriction of $f$ on $U$ is convex, it is easily seen that, for all $t \in] 0,1], x \in \Omega$ and $v \in V$, we have

$$
-f(x-v)+f(x) \leqslant t^{-1}(f(x+t v)-f(x)) \leqslant f(x+v)-f(x) .
$$

For each $v \in E$ and each $x \in E$ put

$$
a(v)=-f(\bar{x}-v)+f(\bar{x}) \quad \text { and } \quad b(v)=f(\bar{x}+v)-f(\bar{x})
$$

and

$$
\begin{aligned}
& \eta(x, v)=\inf (0,-f(x-v)+f(x)+f(\bar{x}-v)-f(\bar{x})) \\
& \gamma(x, v)=\sup (0, f(x+v)-f(x)-f(\bar{x}+v)+f(\bar{x})) .
\end{aligned}
$$

Then, since $F$ is a vector lattice, according to corollary 1.4, chap. 1 in [32], we have

$$
[a(v)+\eta(x, v), b(v)+\gamma(x, v)]=[a(v), b(v)]+[\eta(x, v), \gamma(x, v)]
$$


and hence there exist, for each $t \in] 0,1]$, each $x \in \Omega$ and each $v \in V$, an element

$$
d(t, x ; v) \in[\eta(x, v), \gamma(x, v)]
$$

such that

$$
t^{-1}(f(x+t v)-f(x)) \in[a(v), b(v)]+d(t, x ; v) .
$$

Define a mapping $K$ of $E$ into $\operatorname{Comp}\left(F_{\theta}\right)$ and a mapping $r$ of $\left.] 0,1\right] \times E \times E$ into $F$ by setting

$$
K(v)=[a(v), b(v)] \quad \text { for each } v \in E,
$$

and

$$
\begin{array}{ll}
r(t, x ; v)=d(t, x ; v) & \text { if } x \in \Omega \text { and } v \in V \\
r(t, x ; v)=0 & \text { otherwise. }
\end{array}
$$

Since the mapping $f$ is continuous on $U$, that the lattice operations are continuous for the topology of $F$ and that the positive cone $F_{+}$is normal, it is not difficult to verify that the multifunction $K$ is upper-semicontinuous with $K(0)=\{0\}$,

$$
\lim _{\substack{t \downarrow 0 \\ x \rightarrow \bar{x}}} r(t, x ; v)=0 \quad \text { for each } v \in V \quad \text { and } \quad \lim _{\substack{t \downarrow 0 \\ x \rightarrow \bar{x} \\ v \rightarrow 0}} r(t, x ; v)=0 \text {. }
$$

So conditions $\left(a^{\prime}\right),\left(b^{\prime}\right)$ and $\left(c^{\prime}\right)$ of definition 1.1 are verified, and the proposition is proved.

REMARK. - The above proof shows that the proposition still holds if the mapping $x \mapsto \sup (x, 0)$ of $F$ into $F_{\theta}$ is continuous and if the positive cone $F_{+}$is normal with respect to the $\theta$-topology.

\section{2. - Generalized directional derivative of a compactly Lipschitzian mapping.}

In this section and the following ones we shall denote by $E$ a topological vector space and by $F$ an ordered topological vector space. We shall assume that $F$ is also an order-complete vector lattice for its order structure, that is, $\sup (x, y)$ exists for all $x, y \in F$ and $\sup M$ exists for each nonempty subset $M$ of $F$ which is bounded above in $F$ (see [32]).

By $F^{*}=F \cup\{+\infty\}$ we shall mean the order space $F$ with the adjunction of a supremum $+\infty$. It follows that every nonempty subset of $F$ has a supremum in $F^{*}$. 
Let $f$ be a mapping of $E$ into $F$ which is compactly lipsehitzian at a point $\bar{x} \in E$. Consider the following mapping

$$
\begin{aligned}
& \left.q_{f}:\right] 0,+\infty[\times E \times E \rightarrow F \\
& q_{f}(t, x ; v)=t^{-1}[f(x+t v)-f(x)] .
\end{aligned}
$$

For every subset $M$ of $F$ we shall denote by $\mathrm{cl}_{F}(M)$ the closure of $M$ in $F$. We shall put:

$$
D_{f}(\bar{x} ; v)=\bigcap_{\varepsilon, W} \mathrm{cl}_{F}\left[q_{f}(] 0, \varepsilon[\times W ; v)\right]
$$

where $W$ is a neighborhood of $\bar{x}$ in $E$ and $\varepsilon$ is a positive real number.

We remark that $D_{f}(\widetilde{x} ; v)$ is also the set of limits of all convergent nets $\left(q_{f}\left(t_{j}, x_{j} ; v\right)\right)_{j \in J}$ such that $\left(t_{j}\right)_{j \in J}$ is a net of positive real numbers converging to zero and $\left(x_{j}\right)_{j \in J}$ is a net in $E$ converging to $\bar{x}$.

Hence, we may write

$$
D_{f}(\bar{x} ; v)=\left\{\lim _{j \in J} q_{f}\left(t_{j}, x_{j} ; v\right) \mid t_{j}>0, \lim _{j \in J} t_{j}=0, \lim _{j \in J} x_{j}=\bar{x}\right\} .
$$

Since $f$ is compactly lipsehitzian at $\bar{x}$, it is easily seen that $D_{f}(\bar{x} ; v) \neq \emptyset$ for every $v \in E$.

2.1. Definition. - The mapping $f^{0}(\vec{x} ; \cdot)$ of $E$ into $F^{\bullet}$ defined by $f^{0}(\vec{x} ; v)=$ $=\sup D_{f}(\bar{x} ; v)$ for every $v \in E$ will be called, following Clarke's terminology, the generalized directional derivative of $f$ at the point $\bar{x}$.

REMARK. - It is easily seen that, if $E$ is a normed vector space and if $f$ is a real valued function which is Lipschitz at a point $\bar{x}, f^{0}(\bar{x} ; \cdot)$ coincides with Clarke's directional derivative (CLARKe $[6,7]$ ).

2.2. Proposimion. - Let $\lambda$ be a nonnegative real number and let $f, g$ be two mappings of $E$ into $F$ which are compactly lipschitzian at a point $\bar{x}$. Then for every $v \in E$, the following assertions hold:

a) $(\lambda f)^{\circ}(\bar{x} ; v)=\lambda f^{0}(\bar{x} ; v)$;

b) $(-f)^{0}(\bar{x} ; v)=f^{0}(\bar{x} ;-v)$;

c) $(f+g)^{0}(\widetilde{x} ; v) \leqslant f^{0}(\bar{x} ; v)+g^{0}(\bar{x} ; v)$ :

Proof. - Naking use of the obvious relations $q_{\lambda_{f}}=\lambda q_{f}$ and $q_{f_{f}}=q_{f}+g_{g}$, we get the following:

$$
D_{\lambda r}(\bar{x} ; v)=\lambda D_{f}(\bar{x} ; v) \quad \text { and } \quad D_{f ; g}(\bar{x} ; v) \subset D_{f}(\bar{x} ; v)+D_{g}(\bar{x} ; v) \text {. }
$$


Hence, the assertions $(a)$ and $(c)$ are verified. Let us observe now that we have:

$$
\begin{aligned}
q_{-f}(t, x ; v) & =-t^{-1}[f(x+t v)-f(x)] \\
& =t^{-1}[f((x+t v)+t(-v))-f(x+t v)] \\
& =q_{f}(t, x+t v ;-v) .
\end{aligned}
$$

Let $W$ be a neighborhood of $\bar{x}$ in $E$ and let $\varepsilon$ be a positive real number. We claim that $W+] 0, \varepsilon[v$ is a neighborhood of $\bar{x}$ in $E$. Indeed, since the mapping $(x, t) \mapsto x+t v$ of $E \times \mathbb{R}$ into $E$ is continuous, there exists a real number $\eta$ with $0<\eta<\varepsilon$ and a neighborhood $U$ of $\bar{x}$ in $E$ such that

$$
U-] 0, \eta[v \subset U+]-\eta, \eta[v \subset W
$$

Therefore, we have

$$
U \subset W+] 0, \eta[v \subset W+] 0, \varepsilon[v .
$$

Using this fact and the definition of $D_{-f}(\bar{x} ; v)$, we see that the following inclusion holds:

$$
D_{-f}(\bar{x} ; v) \subset D_{f}(\bar{x} ;-v) \text {. }
$$

By symmetry we obtain that the reverse inclusion holds too. Hence, we may conclude that we have:

$$
(-f)^{0}(\bar{x} ; v)=\sup D_{-f}(\bar{x} ; v)=\sup D_{f}(\bar{x} ;-v)=f^{0}(\bar{x} ;-v) .
$$

The following proposition gives a very important property of the generalized directional derivative.

2.3. Proposition. - If $f$ is compactly lipschitzian at a point $\bar{x} \in E$, then the mapping $f^{0}(\bar{x} ; \cdot)$ of $E$ into $F^{\bullet}$ is sublinear.

\section{Proof.}

1) If $\lambda=0$, it is obvious that $f^{0}(\bar{x} ; \lambda v)=\lambda f^{0}(\bar{x} ; v)$ for every $v \in E$. Let $\lambda$ be a positive real number and let $v$ be a point in $E$. Since $q_{f}(t, x ; \lambda v)=\lambda q_{f}(\lambda t, x ; v)$, if we denote by $\mathfrak{B}(\bar{x})$ the set of neighborhoods of $\bar{x}$, we have:

$$
\begin{aligned}
D_{f}(\bar{x} ; \lambda v) & =\bigcap_{\substack{\varepsilon>0 \\
W \in \mathfrak{B}(\bar{x})}} \operatorname{cl}_{F}\left[q_{f}(] 0, \varepsilon[\times W ; \lambda v)\right] \\
& =\lambda \bigcap_{\substack{\varepsilon>0 \\
W \in \mathfrak{B}(\bar{x})}} \mathrm{cl}_{F}\left[q_{f}(] 0, \lambda \varepsilon[\times W ; v)\right] \\
& =\lambda \bigcap_{\substack{\varepsilon>0 \\
W \in \mathfrak{B}(\bar{x})}} \operatorname{cl}_{F^{*}}\left[q_{f}(] \mathbf{0}, \varepsilon[\times W ; v)\right] \\
& =\lambda D_{f}(\bar{x} ; v) .
\end{aligned}
$$

Thus, we have $f^{0}(\bar{x} ; \lambda v)=\lambda f^{0}(\bar{x} ; v)$. 
2) Let $v$ and $w$ be two points in $E$. Choose a mapping $K$ of $E$ into Comp (F) and a mapping $r$ of $] 0,1] \times E \times E$ into $F$ verifying the properties of definition 1.1, and note that we have

$$
q_{f}(t, x ; v+w)=q_{f}(t, x ; v)+q_{f}(t, x+t v ; w)
$$

for every positive real number $t$ and for every $x \in E$. Now consider a point $\lim _{j \in J} q_{f}\left(t_{i}, x_{j} ; v+w\right)$ in $D_{f}(\bar{x} ; v+w)$. There exists an element $j_{0} \in J$ such that

$$
q_{f}\left(t_{j}, x_{j} ; v\right)-r\left(t_{j}, x_{j} ; v\right) \in K(v)
$$

and

$$
q_{j}\left(t_{j}, x_{j}+t_{j} v ; w\right)-r\left(t_{j}, x_{j}+t_{j} v ; w\right) \in K(w)
$$

for every $j \in J$ verifying $j \geqslant j_{0}$. Hence, making use of the relation $\lim _{\substack{t \rightarrow 0 \\ x \rightarrow \overline{\bar{x}}}} r(t, x ; z)=0$ for every $z \in E$, we can find two convergent subnets

$$
\left(q_{f}\left(t_{\alpha(i)}, x_{\alpha(i)} ; v\right)\right)_{i \in I} \quad \text { and } \quad\left(q_{f}\left(t_{\alpha(i)}, x_{\alpha(i)}+t_{\alpha(i)} v ; w\right)\right)_{i \in I} .
$$

Therefore, we may conclude that

$$
D_{f}(\bar{x} ; v+w) \subset D_{f}(\bar{x} ; v)+D_{f}(\bar{x} ; w)
$$

and

$$
f^{0}(\bar{x} ; v+w) \leqslant f^{0}(\bar{x} ; v)+f^{0}(\bar{x} ; w) .
$$

In order to give a class of mappings for which the generalized directional derivative is a continuous mapping, we consider the following lemma.

2.4. Leima. - Assume that the positive cone $F_{+}$is normal. If $s$ is a sublinear mapping of $E$ into $F$ which is continuous at the origin, then $s$ is continuous on $E$.

Proof. - Let $x_{0}$ be a point in $E$. Noting that $-s\left(x_{0}-x\right) \leqslant s(x)-s\left(x_{0}\right) \leqslant s\left(x-x_{0}\right)$ for every $x \in E$ and using the normality of the positive cone $F_{+}$and the equalities

$$
\lim _{x \rightarrow x_{0}} s\left(x-x_{0}\right)=\lim _{x \rightarrow x_{0}}\left[-s\left(x_{0}-x\right)\right]=0,
$$

we may conclude (see 1.7) that $\lim _{x \rightarrow x_{0}} s(x)=s\left(x_{0}\right)$.

2.5. Definition. - A mapping $f$ of a topological vector space $E$ into an ordered topological vector space $F$ will be said to be order Lipsehitz at a point $\bar{x} \in E$ if there 
exist two mappings $\underline{h}$ and $\bar{h}$ of $E$ into $F$ and a mapping $r$ of $] 0,1] \times E \times E$ into $F$ such that

a) $\underline{h}(v) \leqslant \bar{h}(v)$ for all $v \in E$ and $\lim _{v \rightarrow 0} \bar{h}(v)=0$;

b) $\lim r(t, x ; v)=0$ for all $v \in E$; $\underset{x \rightarrow \infty}{t \downarrow 0}$

c) for each $v \in E$ there is a neighborhood $\Omega$ of $\bar{x}$ in $E$ and a real number $\eta \in] 0,1]$ for which

$$
t^{-1}(f(x+t v)-f(x)) \in[\underline{h}(v), \bar{h}(x)]+r(t, x ; v)
$$

for all $t \in] 0, \eta]$ and $x \in \Omega$.

REMark. - Consider a normed vector space $E$ and a normed vector lattice $F$. Assume that $f$ is a mapping of $E$ into $F$ and $\bar{x}$ is a point of $E$ such that there exist a point $k \in F_{+}$and a neighborhood $\Omega$ of $\bar{x}$ satisfying

$$
f(x)-f(y) \leqslant\|x-y\| k
$$

for all $x, y$ in $\Omega$. If we denote by $|\cdot|$ the absolute value mapping of $F$, then we have

$$
|f(x)-f(y)| \leqslant\|x-y\| k
$$

for all $x, y$ in $\Omega$. Then the mapping $f$ is Lipschitz at the point $\bar{x}$, since we have

$$
\|f(x)-f(y)\| \leqslant\|k\| \cdot\|x-y\|
$$

(in a normed vector lattice the inequality $\left|z_{1}\right| \leqslant\left|z_{2}\right|$ implies $\left\|z_{1}\right\| \leqslant\left\|z_{2}\right\|$, see [32]).

2.6. Proposition. - Assume that the positive cone $\vec{F}_{+}$is normal and that the order intervals are compact in $F$. If $f$ is order Lipschitz at a point $\bar{x} \in E$, then $f$ is compactly lipschitzian at $\bar{x}$ and $f(\bar{x} ; \cdot)$ is a continuous sublinear mapping of $E$ into $F$.

Proof. - Choose a mapping $\bar{h}$ of $E$ into $F$ such that $\lim _{v \rightarrow 0} \bar{h}(v)=0$ and a mapping $r$ of $] 0,1] \times E \times E$ into $F$ verifying the conditions of definition 2.5 . It is obvious that $f$ is compactly lipschitzian since the order intervals are compact in $F$.

If $v$ is a point in $E$ and if $\lim _{j \in J} q_{f}\left(t_{j}, x_{j} ; v\right)$ is a point in $D_{f}(\bar{x} ; v)$, then with the help of the above facts it is easily seen that $\lim _{j \in J} q_{f}\left(t_{j}, x_{j} ; v\right) \leqslant \vec{h}(v)$. Hence we have $-\bar{h}(-v) \leqslant f^{\circ}(x ; v) \leqslant \breve{h}(v)$ for every $v \in E$. Therefore, the normality of $F_{+}$and the relation $\lim _{v \rightarrow 0} \bar{h}(v)=0$ imply that $f^{0}(\bar{x} ; \cdot)$ is continuous at the origin. Making use of the above lemma we conclude that $f^{0}(x ; \cdot)$ is continuous on $E$.

As a consequence of the above proposition we have the following result. 
2.7. Proposition. - Under the assumptions of the above proposition, the mapping $f^{0}(\bar{x} ; \cdot)$ of $E$ into $F$ is order Lipschitz on $E$.

Proof. - In the proof of the preceding proposition we have seen that the relation $f^{0}(\bar{x} ; \cdot) \leqslant \bar{h}$ holds. Therefore, if we assume that $v$ and $w$ are two points in $E$, we conclude that we have:

$$
f^{0}(\bar{x} ; v)-f^{0}(\bar{x} ; w) \leqslant f^{0}(\bar{x} ; v-w) \leqslant \bar{h}(v-w) .
$$

REMARK. - More generally, every continuous sublinear mapping is order lipschitz on $E$. It is obvious that the continuity of the mapping $f^{0}(\bar{x} ; \cdot)$ is very important. So we introduce the following definition.

2.8. Definition. - We shall say that a point $\bar{x}$ is regular for a mapping $f$ of $E$ into $F$ if $f$ is compactly lipschitzian at $\bar{x}$ and if $f^{0}(\bar{x} ; \cdot)$ is a continuous mapping of $E$ into $F$. Sometimes we shall say that $f$ is regular at $\bar{x}$.

\section{REMARKS.}

1) Obviously, if $\bar{x}$ is a regular point for $f$, the mapping $f^{0}(\bar{x} ; \cdot)$ is then convex and continuous.

2) If the cone $F_{+}$is normal and if the multifunction $K$ in definition 1.1 is bounded above on a neighborhood of zero, then it is easily seen that $\bar{x}$ is a regular point for $f$.

\section{3. - Subdifferential of a compactly Lipschitzian mapping.}

$L\left(E, F^{\prime}\right)$ will denote the vector space of all continuous linear mappings of $E$ into $F$ and $L_{s}(E, F)$ this space endowed with the topology of pointwise convergence.

3.1. Definitions. - Let $f$ be a mapping of $E$ into $F$ which is compactly lipschitzian at a point $\bar{x} \in E$. An element $T \in L(E, F)$ will be called a subgradient of $f$ at $\bar{x}$ if the following relation holds:

$$
T(v) \leqslant f^{0}(\bar{x} ; v) \quad \text { for all } v \in E .
$$

The set of all subgradients of $f$ at $\bar{x}$ will be called the subdifferential of $f$ at $\bar{x}$ and denoted by $\partial f(\bar{x})$.

\section{REMARKs.}

1) The subdifferential $\partial f(\bar{x})$ can be empty. It is the case if $f$ is a noncontinuous linear mapping since $f^{\circ}(\overline{\bar{x}} ; v)=f(v)$ for all $v \in E$.

2) If $f$ is a real-valued function which is Lipschitz at $\bar{x}$ and if $E$ is normed, then the above subdifferential is exactly the one of ClarKE $[6,7]$. 
3.2. Proposition. - Assume that $\bar{F}_{+}$is a normal cone and that $\bar{x}$ is a regular point for the mapping $f$. Then the subdifferential $\partial f(\bar{x})$ of $f$ at $\bar{x}$ is the set of all linear mappings $T$ of $E$ into $F$ such that $T(v) \leqslant f^{0}(\bar{x} ; v)$ for all $v \in E$.

Proof. - Suppose that $T$ is a linear mapping of $E$ into $F$ verifying the above assumption. Since $f^{0}(\bar{x} ; \cdot)$ is a sublinear mapping of $E$ into $F$, we have $-f^{0}(\bar{x} ;-v) \leqslant$ $\leqslant T(v) \leqslant f^{0}(\bar{x} ; v)$. Using the normality of $F_{+}$, we conclude that $\lim _{v \rightarrow 0} T(v)=0$, and hence $T$ is continuous on $E$.

REMARK. - The relation $T(v) \in\left[-f^{0}(\bar{x} ;-v), f^{0}(\bar{x} ; v)\right]$ for every $T \in \partial f(\bar{x})$ implies that $\partial f(\bar{x})$ is an equicontinuous subset of $L(E, F)$ when the assumptions of the above proposition are verified. Indeed, let $W$ be any neighborhood of the origin in $F$ verifying $W=\left(W+F_{+}\right) \cap\left(W-F_{+}\right)$. Since the mappings $v \mapsto-f^{0}(\bar{x} ;-v)$ and $v \mapsto$ $\nrightarrow f^{0}(\bar{x} ; v)$ are continuous, there exists a neighborhood $V$ of the origin in $E$ for which we have $-f^{0}(\bar{x} ;-v) \in W$ and $f^{0}(\bar{x} ; v) \in W$ for every $v \in V$. Thus we may conclude that $T(V) \subset W$ for all $T \in \partial f(\bar{x})$.

Below we shall make use of a generalization of the analytical form of the HahnBanach Theorem (see [21] or [32]).

3.3. LEMMA. - Let $s$ be a sublinear mapping of a real vector space $X$ into an order complete vector lattice $Y$. If $T$ is a linear mapping defined on a vector subspace $H$ of $X$ with range in $Y$ verifying the relation $T(x) \leqslant s(x)$ for all $x \in H$, then $T$ can be extended to a linear mapping $\bar{T}$ of $X$ into $Y$ such that $\bar{T}(x) \leqslant s(x)$ for all $x \in X$.

3.4. PRopostrion. - Under the assumptions of proposition 3.2, the subdifferential $\partial f(\bar{x})$ is a nonempty convex closed subset of $L_{s}(E, F)$ and

$$
f^{0}(\bar{x} ; v)=\max \{T(v) \mid T \in \partial f(\bar{x})\} \quad \text { for all } v \in E .
$$

Moreover, $\partial f(\bar{x})$ is a compact subset of $L_{s}(E, F)$ if the order intervals are compact in $F$.

Proof. - We may assume that $E \neq\{0\}$ and let $v$ be an element of $E$ different from the origin. The linear mapping $T$ of $\mathbb{R} \cdot v$ into $F$ defined by $T(t v)=t f^{0}(\bar{x} ; v)$ for all $t \in \mathbb{R}$ verifies the relation $T(t v) \leqslant f^{0}(\bar{x} ; t v)$. If $t$ is non-negative, the inequality is obvious. Suppose that $t$ is negative. Since the relation $0 \leqslant f^{0}(\bar{x} ; v)+f^{0}(\vec{x} ;-v)$ holds, we have:

$$
\begin{aligned}
T(t v) & =(-t)\left(-f^{0}(\bar{x} ; v)\right) \\
& \leqslant-t f^{0}(\bar{x} ;-v) \\
& =f^{0}(\bar{x} ; t v) .
\end{aligned}
$$

The above lemma asserts that there exists a linear mapping $\bar{T}$ of $E$ into $F$ extending $T$ such that $\bar{T}(y) \leqslant f^{0}(\bar{x} ; y)$ for all $y \in E$. Proposition 3.2 implies that $\bar{T} \in \partial f(\bar{x})$. 
Therefore, we have $\partial f(\bar{x}) \neq \emptyset$ and $f^{0}(\bar{x} ; v)=\max \{A(v) \mid A \in \partial f(\bar{x})\}$. To prove that $\partial f(\vec{x})$ is a closed subset of $L_{s}(E, F)$, consider for each $v \in E$ the mapping $l_{v}$ of $L_{s}(E, F)$ into $F$ defined by $l_{v}(T)=f^{0}(\bar{x} ; v)+T(-v)$. It is continuous. Since $F_{+}$is a closed subset of $F, \partial f(\bar{x})=\bigcap_{v \in E} l_{v}^{-1}\left(F_{+}\right)$is a closed subset of $L_{s}(E, F)$. As the convexity of $\partial f(\bar{x})$ is obvious, it remains to show that $\partial f(\bar{x})$ is a compact subset of $L_{s}\left(E, F^{\prime}\right)$ if the order intervals are compact in $F$. Since $\partial f(\bar{x})$ is equicontinuous, it suffices to note that we have $T(v) \in\left[-f^{0}(\bar{x} ;-v), f^{0}(\bar{x} ; v)\right]$ for all $T \in \partial f(\bar{x})$ and $v \in E$ to conclude that $\partial f(\bar{x})$ is relatively compact in $L_{s}(E, F)$ hence compact.

\section{REMARKS.}

1) The above arguments are close to the ones used by VALADIER in [43]. Similar results have been also obtained by IOFFE and Levin [15] and ZowE [45] for convex functions and PENOT [30] for unscarped, tangentially convex functions.

2) By the Ascoli theorem, instead of the pointwise convergence topology, we may also consider the compact or precompact convergence topology on $L(E, F)$ in the above results.

3) If $F$ is a semi-reflexive locally convex space and if the positive cone $F_{+}$ is normal, the order intervals are weakly compact in $F$ since they are bounded and closed convex subsets of $F$.

The following proposition gives an expected result.

3.5. Proposition. - Let $f$ be a mapping of $E$ into $F$ which is compactly lipschitzian at a point $\bar{x} \in E$. If the point $\bar{x}$ is a local minimum for the mapping $f$, then we have $0 \in \partial f(\bar{x})$.

Proor. - Choose $K$ and $r$ verifying the conditions of definition 1.1. We may assume that $f(\vec{x}) \leqslant f(x)$ for all $x \in \Omega$, where $\Omega$ is a neighborhood of $\bar{x}$ in $E$.

Consider a point $v \in E$ and a net $\left(t_{j}\right)_{j \in J}$ of $\left.] 0,1\right]$ converging to zero. For some $j_{0} \in J$ we have $q_{f}\left(t_{j}, \bar{x} ; v\right)-r\left(t_{j}, \vec{x} ; v\right) \in \bar{K}(v)$ and $\bar{x}+t_{j} v \in \Omega$ for all $j \geqslant j_{0}$. Also, there exists a convergent subnet $\left(q_{f}\left(t_{\alpha(i)}, \bar{x} ; v\right)\right)_{i \in I}$. Therefore, the inequalities

$$
0 \leqslant \lim _{i \in I} q_{f}\left(t_{\alpha(i)}, \bar{x} ; v\right) \leqslant f^{0}(\bar{x} ; v)
$$

are verified, and hence we conclude that $0 \in \partial f(\bar{x})$.

The subdifferential has the following property.

3.6. Proposition. - Let $f$ be a mapping of $E$ into $F$ which is compactly lipschitzian at a point $\bar{x} \in E$. The formula $\partial(-f)(\bar{x})=-\partial f(\bar{x})$ holds.

PRoof. - It is a direct consequence of the assertion of proposition 2.2 which says that $(-f)^{0}(\bar{x} ; v)=f^{0}(\bar{x} ;-v)$ for every $v \in E$.

To close this section, we now proceed to show that, if $f$ is compactly lipsehitzian at a point $\bar{x} \in E$, there exists a convex mapping of $E$ into $F$ such that its convex 
subdifferential at the origin coincides with $\partial f(\bar{x})$, and to study the case where $f$ is convex.

Let us recall that a mapping $g$ of $E$ into $F^{\bullet}$ is said to be convex if for all $x, y \in E$ and real numbers $t \in[0,1]$

$$
g(t x+(1-t) y) \leqslant t f(x)+(1-t) f(y)
$$

We need also some further definitions. The domain of $g$ is the set dom $g=$ $=\{x \in E \mid g(x) \in F\}$. If $x_{0}$ is a point in dom $g$, the set of all elements $T$ of $L(E, F)$ such that

$$
T(x)-T\left(x_{0}\right) \leqslant g(x)-g\left(x_{0}\right) \quad \text { for all } x \in E
$$

is the convex subdifferential of $g$ at $x_{0}$. It will be denoted by $\partial_{e} g\left(x_{0}\right)$.

We shall put (see [43]) $f^{\prime}\left(x_{0} ; v\right)=\inf _{t>0} t^{-1}\left[f\left(x_{0}+t v\right)-f\left(x_{0}\right)\right]$ for all $v \in E$.

3.7. Proposition. - Let $f$ be a mapping of $E$ into $F$ which is compactly lipschitzian at a point $\bar{x} \in E$. Then the mapping $f^{0}(\bar{x} ; \cdot)$ of $E$ into $F^{\bullet}$ is convex and the following equality holds:

$$
\partial f(\bar{x})=\partial_{c} f^{0}(\bar{x} ; \cdot)(0)
$$

Proof. - The convexity of the mapping $f^{0}(\bar{x} ; \cdot)$ is trivially verified and $0 \in$ $E \operatorname{dom} f^{0}(\vec{x} ; \cdot)$ since $f^{0}(\bar{x} ; 0)=0$. Moreover, a continuous linear mapping $T$ of $E$ into $F$ is in $\partial f(\bar{x})$ if and only if $T(v) \leqslant f^{\circ}(\bar{x} ; v)$ for all $v \in E$ (see the definition of $\partial f(\bar{x}))$ and hence if and only if it is in $\partial_{c} f^{0}(\bar{x} ; \cdot)(0)$.

The following three lemmas will allow us to compare the lipschitzian subdifferential with the convex one for a convex mapping.

3.8. LEMnA. - If $\left(x_{j}\right)_{j \in J}$ is a decreasing net whose elements are in a compact subset $K$ of an order topological vector $X$, then this net is convergent and we have

$$
\lim _{j \in J} x_{j}=\inf _{j \in J} x_{j}
$$

Proof. - Since $K$ is compact, it suffices to prove that any cluster point $y$ of the net $\left(x_{j}\right)_{j \in J}$ is the infimum of the family $\left\{x_{j} \mid j \in J\right\}$. For each $j \in J$, since $S_{j}=\left\{x_{k} \mid k \in J\right.$, $k \geqslant j\} \subset x_{j}-X_{+}$and that the positive cone $X_{+}$is closed, we have $y \in \operatorname{cl}\left(S_{j}\right) \subset x_{j}-X_{+}$. Therefore, for each $j \in J$ we have $y \leqslant x_{j}$. If a point $z \in X$ verifies $z \leqslant x_{j}$ for all $j \in J$, we have $z \leqslant y$, for $y$ is a cluster point of the net $\left(x_{j}\right)_{j \in J}$ and hence $y=\inf _{j \in J} x_{j}$.

3.9. LEMnMA. - Let $G$ be an order-complete vector lattice endowed with a topology for which it is an ordered topological vector space. If a mapping $f$ of $E$ into $E$ is convex 
and compactly lipschitzian at a point $\vec{x} \in E$, then for every continuous positive linear mapping $A$ of $F$ into $G$ we have

$$
(A \circ f)^{\prime}(\vec{x} ; v)=A\left(f^{\prime}(\bar{x} ; v)\right) \quad \text { for every } v \in E .
$$

Proof. - Let $K$ be a mapping of $E$ into Comp $(F), r$ a mapping of $] 0,1 \times E \times E$ into $F$ verifying the conditions of definition 1.1. Let $v$ be any point in $E$. There exist a neighborhood $\Omega$ of $\bar{x}$ in $E$ and a real number $\eta \in] 0,1]$ such that $q_{f}(t, x ; v) \in$ $\in K(v)+r(t, x ; v)$ for all $(t, x) \in] 0, \eta] \times \Omega$. Since $f$ is convex, the mappings $q_{f}(\cdot, \bar{x} ; v)$ and $q_{A \text { of }}(\cdot, \vec{x} ; v)$ defined on the real interval $] 0,+\infty[$ are increasing. Therefore, if we consider a decreasing sequence $\left(t_{n}\right)_{n \in \mathbb{N}}$ in $\left.] 0, \eta\right]$ converging to zero we have

$$
f^{\prime}(\bar{x} ; v)=\inf _{n \in \mathbb{N}} q_{f}\left(t_{n}, \bar{x} ; v\right) \quad \text { and } \quad(A \circ f)^{\prime}(\bar{x} ; v)=\inf _{n \in \mathbb{N}} q_{A \circ f}\left(t_{n}, \bar{x} ; v\right)
$$

But if we put $R=\left\{r\left(t_{n}, \bar{x} ; v\right) \mid n \in \mathbb{N}\right\} \cup\{0\}$, the decreasing sequences $\left(q_{f}\left(t_{n}, x ; v\right)\right)_{n \in \mathbb{N}}$ and $\left(q_{A \text { of }}\left(t_{v}, \bar{x} ; v\right)\right)_{n \in \mathbb{N}}$ are respectively in the compact $K(v)+R$ and $A(K(v))+A(R)$. Thus the preceding lemma ensures that

$$
(A \circ f)^{\prime}(\bar{x} ; v)=\lim _{n \rightarrow \infty} q_{\Delta \circ f}\left(t_{n}, \bar{x} ; v\right)=\lim _{n \rightarrow \infty} A\left(q_{f}\left(t_{n}, \bar{x} ; v\right)\right)=A\left(f^{\prime}(\bar{x} ; v)\right)
$$

and the proof is complete.

3.10. LeMrM. - Let $g$ be a convex real-valued function defined on $E$. If $g$ is continuous at $\bar{x} \in E$ then $g$ is compactly lipschitzian at $\bar{x}$ and we have

$$
g^{0}(\bar{x} ; v)=g^{\prime}(\bar{x} ; v) \quad \text { for all } v \in E \text {. }
$$

Proof. - In proposition 1.9 we have seen that $g$ is compactly lipsehitzian at $\vec{x}$. Let $v$ be any point in $E$. The inequality $g^{\prime}(\bar{x} ; v) \leqslant g^{0}(\bar{x} ; v)$ being obvious, let us show the reverse inequality. We shall follow the proof of proposition 3 of ClaRkE [7]. Let $\lim _{j \in J} q_{g}\left(t_{j}, x_{j} ; v\right)$ be any point in $D_{g}(\bar{x} ; v)$. Since $g$ is continuous, the mean value theorem for convex real-valued functions implies that, for each $j \in J$, there exist $y_{j}=\left(1-s_{j}\right) x_{j}+s_{j}\left(x_{j}+t_{j} v\right)$ with $\left.s_{j} \in\right] 0,1\left[\right.$ and $z_{j} \in \partial_{c} g\left(y_{j}\right)$ verifying

$$
q_{g}\left(t_{j}, x_{j} ; v\right)=\left\langle z_{j}, v\right\rangle \leqslant g^{\prime}\left(y_{j} ; v\right) .
$$

Since the function $x \mapsto g^{\prime}(x ; v)=\inf _{t>0} t^{-1}[g(x+t v)-g(x)]$ is upper semi-continuous, we obtain that

$$
\lim _{j \in J} q_{g}\left(t_{j}, x_{j} ; v\right) \leqslant \limsup _{j \in J} g^{\prime}\left(y_{j} ; v\right) \leqslant g^{\prime}(\bar{x} ; v)
$$

and hence $g^{0}(\bar{x} ; v) \leqslant g^{\prime}(\vec{x} ; v)$. 
3.11. Proposition. - If the space $F$ is locally convex and if $f$ is a convex mapping of $E$ into $F$ which is compactly lipschitzian and continuous at a point $\vec{x} \in E$, then we have $f^{\prime}(\bar{x} ; v)=f^{0}(\vec{x} ; v)$ for all $v \in E$.

Proof. - It is clear from the definitions of $f^{\prime}(\bar{x} ; \cdot)$ and $f^{0}(\bar{x} ; \cdot)$ that $f^{\prime}(\vec{x} ; v) \leqslant f^{0}(\bar{x} ; v)$ for all $v \in E$. Let us show the reverse inequality.

Let $v$ be any point in $E$. Let us consider any point $y^{\prime} \in F^{\prime}$ and any point $\lim _{j \in J} q_{f}\left(t_{j}, x_{j} ; v\right)$ in $D_{f}(\bar{x} ; v)$. Since the convex real-valued function $y^{\prime}$ of is continuous at $\bar{x}$ and hence continuous on $E$, we have $\left(y^{\prime} \circ f\right)^{\prime}(\bar{x} ; v)=\left(y^{\prime} \circ f\right)^{0}(\bar{x} ; v)$ according to lemma 3.10. Then it follows from the continuity of $y^{\prime}$ and lemma 3.9 that we have

$$
y^{\prime}\left(\lim _{j \in J} q_{f}\left(t_{j}, x_{j} ; v\right)\right)=\lim _{j \in J} q_{y^{\prime} \circ f}\left(t_{j}, x_{j} ; v\right) \leqslant\left(y^{\prime} \circ f\right)^{0}(\bar{x} ; v)=y^{\prime}\left(f^{\prime}(\bar{x} ; v)\right) .
$$

Therefore, since $F$ is locally convex, the Hahn-Banach theorem implies that $\lim _{j \in J} q_{f}\left(t_{j}, x_{j} ; v\right) \leqslant f^{\prime}(\vec{x} ; v)$. Thus we may conclude that $f^{0}(\bar{x} ; v) \leqslant f^{\prime}(\bar{x} ; v)$.

An immediate consequence of the above proposition is the following.

3.12. CoRollary. - Under the above assumptions the lipschitzian and convex subdifferentials of $f$ at the point $\bar{x}$ coincide.

\section{4. - Some formulas relative to the Lipschitzian subdifferential.}

Below we shall need the following lemma.

4.1. LEMMA. - Let $g_{1}$ and $g_{2}$ be two convex mappings of $E$ into $F^{\bullet}$ such that $g_{1} \leqslant g_{2}$. Suppose that $x_{0}$ is a point of $E$ verifying $g_{1}\left(x_{0}\right)=g_{2}\left(x_{0}\right) \in F$. Then we have $\partial_{c} g_{1}\left(x_{0}\right) \subset$ с $\partial_{c} g_{2}\left(x_{0}\right)$.

Proof. - This result is a direct consequence of the definition of the convex subdifferential of a convex mapping (see the definitions which follow proposition 3.6).

The following proposition gives a result about the subdifferential of a sum of two compactly lipschitzian mappings.

4.2. Propostiton. - Let $f_{1}$ and $f_{2}$ be two mappings of $E$ into $F$ which are compactly lipschitzian at a point $\bar{x} \in E$ and put $f=f_{1}+f_{2}$. Assume that the positive cone $F_{+}$is normal and that $\bar{x}$ is a regular point for $f_{1}$, then the relation $\partial f(\bar{x}) \subset \partial f_{1}(\bar{x})+\partial f_{2}(\bar{x})$ holds. Moreover, if $\bar{x}$ is also a regular point for $f_{2}$, then $\bar{x}$ is a regular point for $f$ and $\partial f(\bar{x})$ is nonempty.

Proof. - By proposition 2.2 we have $f^{0}(\bar{x} ; v) \leqslant f_{1}^{0}(\bar{x} ; v)+f_{2}^{0}(\bar{x} ; v)$ for all $v \in E$. Since the mapping $f_{1}^{0}(\bar{x} ; \cdot)$ is continuous at the origin, we may apply theorem 4 in Zowe $[46]$ to the convex mappings $f_{1}^{0}(\bar{x} ; \cdot)$ and $f_{2}^{0}(\bar{x} ; \cdot)$. We obtain $\partial_{c}\left(f_{1}^{0}(\bar{x} ; \cdot)+\right.$

12 - Annali di Matematica 
$\left.+f_{2}^{0}(\bar{x} ; \cdot)\right)(0) \subset \partial_{c} f_{1}^{0}(\bar{x} ; \cdot)(0)+\partial_{e} f_{2}^{0}(\vec{x} ; \cdot)(0)$. Hence, using the above lemma and proposition 3.7 , we see that

$$
\partial f(\bar{x})=\partial_{c} f^{0}(\bar{x} ; \cdot)(0) \subset \partial_{c} f_{1}^{0}(\bar{x} ; \cdot)(0)+\partial_{c} f_{2}^{0}(\bar{x} ; \cdot)(0)=\partial f_{1}(\bar{x})+\partial f_{2}(\bar{x}) .
$$

Now suppose that $\bar{x}$ is also a regular point for the mapping $f_{2}$. To prove that $\partial f(\bar{x})$ is non empty, it suffices to prove that $\bar{x}$ is a regular point for the mapping $f$. Since the mapping $f^{0}(\bar{x} ; \cdot)$ is sublinear, the inequalities

$$
-f_{1}^{0}(\bar{x} ;-v)-f_{2}^{0}(\bar{x} ;-v) \leqslant f^{0}(\bar{x} ; v) \leqslant f_{1}^{0}(\bar{x} ; v)+f_{2}^{0}(\bar{x} ; v) \quad \text { hold for each } v \in E .
$$

Then it follows from the normality of the positive cone $F_{+}$and the regularity of $\vec{x}$ for $f_{1}$ and $f_{2}$ that $\lim _{v \rightarrow 0} f^{0}(\bar{x} ; v)=0$. Therefore $f^{0}(\bar{x} ; \cdot)$ is continuous at the origin and hence, by lemma 2.4, continuous on $E$.

\section{REMARKS.}

1) In general the above inclusion cannot be replaced by an equality. It is not even the case when $F$ is the real line and $f_{1}$ and $f_{2}$ are Lipschitz at $\bar{x}$. Indeed, if $g$ is a real function which is Lipschitz at a point $\vec{x}$ and whose subdifferential contains more than one point, take $f_{1}=g$ and $f_{2}=-g$.

2) However, if the mapping $f_{1}$ is strictly differentiable at $\vec{x}$, then it is not diffcult to see that $f^{0}(\bar{x} ; v)=f_{1}^{0}(\bar{x} ; v)+f_{2}^{0}(\bar{x} ; v)$ for all $v \in E$ and hence $\partial f(\bar{x})=\nabla f_{1}(\bar{x})+\partial f_{2}(\bar{x})$.

$\Delta$ bout the subdifferential of a composite mapping $f \circ B$, we have the following relations.

4.3. Proposimion. - Let $B$ be a continuous affine mapping of a topological vector space $G$ into $E$ and let $\bar{x}$ be a point of $G$ such that $B \bar{x}$ is a regular point for a mapping $f$ of $E$ into $F$. If the positive cone $F_{+}$is normal and if $\bar{B}$ denotes the linear mapping associated with $B$, then we have:

i) $\partial(f \circ B)(\bar{x}) \neq \emptyset$ and $\bar{x}$ is a regular point for $f$;

ii) $\partial(f \circ B)(\bar{x}) \subset \partial f(B \bar{x}) \circ \bar{B}$ where $\partial f(B \bar{x}) \circ \bar{B}=\{T \circ \bar{B} \mid T \in \partial f(B \bar{x})\}$.

Proof. - By proposition 1.6 the mapping $f \circ B$ is compactly lipschitzian at $\vec{x}$. Moreover, it is an easy matter to verify that $D_{f \circ B}(\bar{x} ; v) \subset D_{f}(B \bar{x} ; \bar{B} v)$ for all $v \in E$. Then we have $(f \circ B)^{\circ}(\bar{x} ; v) \leqslant f^{0}(B \bar{x} ; \bar{B} v)$ for all $v \in E$. Using an argument similar to that given in the second part of the proof of proposition 4.2, we can say that $\vec{x}$ is a regular point for $f \circ B$ and that $\partial(f \circ B)(\bar{x})$ is nonempty. Now consider an element $A$ of $\partial(f \circ B)(\bar{x})$. By the above inequality we have $-f^{0}(B \bar{x} ;-\bar{B} v) \leqslant A v \leqslant f^{0}(B \bar{x} ; \bar{B} v)$ for all $v \in E$, and hence $A v=0$ whenever $\bar{B} v=0$. Therefore, we can define a linear mapping $T$ of $\operatorname{Im} \bar{B}=\bar{B}(G)$ into $F^{\prime}$ by putting $T(\bar{B} v)=A v$ for all $v \in E$. This linear 
mapping $T$ verifies $T(y) \leqslant f^{0}(B \bar{x} ; y)$ for all $y \in \operatorname{Im} \bar{B}$. By lemma 3.3 we can extend it to a linear mapping $\bar{T}$ of $E$ into $F^{\prime}$ such that $T(y) \leqslant f^{0}(B \bar{x} ; y)$ for all $y \in E$. Finally, it is easily seen that $A=\bar{T} \circ B$ and $\bar{T} \in \partial f(B \bar{x})$.

\section{REMARKS.}

1) If, in addition, the mapping $B$ is open, then, we have $\partial(f \circ B)(\bar{x})=\partial f(B \bar{x}) \circ \bar{B}$. Indeed, let $\lim _{j \in J} q_{f}\left(t_{j}, y_{j} ; \bar{B} v\right)$ be any point in $D_{f}(B \bar{x} ; \bar{B} v)$. For each neighborhood $V$ of the point $\bar{x}$ in $E$, me may choose $j_{V} \in J$ such that $y_{j} \in B(V)$ for all $j \geqslant j_{F}$ since $B(V)$ is a neighborhood of $B \bar{x}$ in $F$. Put $I=\left\{(j, V) \mid V \in \mathfrak{B}(\bar{x}), j \in J, j \geqslant j_{V}\right\}$ where $\mathfrak{B}(\bar{x})$ denotes the filter of neighborhoods of $\bar{x}$ in $E$ and choose for each $(j, V) \in I$ a point $x_{(j, V)} \in V$ with $y_{j}=B x_{(j, V)}$. If we define a preorder relation on $I$ by setting $\left(j_{1}, V_{1}\right) \leqslant$ $\leqslant\left(j_{2}, V_{2}\right)$ if $j_{1} \leqslant j_{2}$ and $V_{1} \supset V_{2}$, it is easily seen that $\left(x_{i}\right)_{i \in I}$ is a net which converges to $\bar{x}$ in $E$. Construct a mapping $\alpha$ of $I$ into $J$ by putting $\alpha(i)=j$ if $i=(j, V)$. We have $y_{\alpha(i)}=B x_{i}$ for every $i \in I$. Consider any point $j_{0} \in J$. If $V_{0}$ is a neighborhood of $\bar{x}$ in $E$ and if we choose $\bar{j} \in J$ verifying $\bar{j} \geqslant j_{0}$ and $\bar{j} \geqslant j_{V_{0}}$, then for $i_{0}=\left(\bar{j}, V_{0}\right)$ we have $\alpha(i) \geqslant j_{0}$ for all $i \geqslant i_{0}$. Thus $\left(y_{\alpha(i)}\right)_{i \in I}$ is a subnet of $\left(y_{j}\right)_{j \in J}$ (see [18]). Therefore, we have

$$
\lim _{j \in J} q_{f}\left(t_{j}, y_{j} ; \bar{B}_{v}\right)=\lim _{i \in I} q_{f}\left(t_{\alpha(i)}, B x_{i} ; \bar{B} v\right)=\lim _{i \in I} q_{f \circ B}\left(t_{\alpha(i)}, x_{i} ; v\right)
$$

and hence $\lim _{j \in J} q_{f}\left(t_{j}, y_{j} ; \bar{B} v\right) \in D_{f \circ B}(\bar{x} ; v)$. Also, we obtain that $f^{0}(B \bar{x} ; \bar{B} v) \leqslant(f \circ B)^{0}(\bar{x} ; v)$ and hence $f^{0}(B \bar{x} ; \bar{B} v)=(f \circ B)^{\circ}(\bar{x} ; v)$. Finally, if $T \in \partial f(B \bar{x})$, then we have

$$
T \circ \bar{B}(w) \leqslant f^{0}(B \bar{x} ; \bar{B} w)=(f \circ B)^{0}(\bar{x} ; w)
$$

for all $w \in E$ and hence $T \circ \bar{B} \in \partial(f \circ B)(\bar{x})$.

2) If $B$ is a surjective and continuous affine mapping of a Fréchet space onto another one, then $B$ is open since the linear mapping associated with it is surjective hence open according to the well known Banach theorem.

4.4. Proposition. - Assume that $E$ is a normed vector space and that the order intervals of $F$ are compact. Let $g$ be a mapping of a normed vector space $G$ into $E$ which is strictly differentiable at a point $\bar{x} \in G$. If $f$ is a mapping of $E$ into $F$ for which there exist a neighborhood $W$ of $g(\bar{x})$ and a point $k \in F_{+}$such that

$$
f\left(y_{1}\right)-f\left(y_{2}\right) \leqslant\left\|y_{1}-y_{2}\right\| k \quad \text { for all } y_{1} \in W \text { and } y_{2} \in W
$$

then the mappings $f$ and fog are respectively compactly lipschitzian at the points $g(\bar{x})$ and $\bar{x}$ and we have

$$
\partial(f \circ g)(\bar{x}) \subset(\partial f(g(\bar{x}))) \circ \nabla g(\bar{x})
$$


Proof. - Since the order intervals are compact in $F$, it follows from example 17.c. that $f$ and $f \circ g$ are respectively compactly lipschitzian at $g(\bar{x})$ and $\bar{x}$. Now, let $v$ be any point in $G$ and let $\lim _{j \in J} q_{f \circ g}\left(t_{j}, x_{j} ; v\right)$ be any point in $D_{f \circ g}(\bar{x} ; v)$. Since $g$ is strictly differentiable at the point $\bar{x}$ and since $f$ verifies the above relation, we have:

$$
\begin{aligned}
\lim _{j \in J} q_{j a g}\left(t_{j}, x_{j} ; v\right) & =\lim _{i \in J} t_{j}^{-1}\left[f\left(g\left(x_{j}\right)+t_{j} \nabla g(\bar{x}) \cdot v+t_{j} \circ\left(t_{j}\right)\right)-f\left(g\left(x_{j}\right)\right)\right] \\
& =\lim _{j \in J} t_{j}^{-1}\left[f\left(g\left(x_{j}\right)+t_{j} \nabla g(\bar{x}) \cdot v\right)-f\left(g\left(x_{j}\right)\right)\right] \\
& =\lim _{j \in J} q_{f}\left(t_{j}, g\left(x_{j}\right) ; \nabla g(\bar{x}) \cdot v\right) .
\end{aligned}
$$

Therefore, we obtain that $(f \circ g)^{0}(\bar{x} ; v) \leqslant f^{0}(g(\bar{x}) ; \nabla g(\bar{x}) \cdot v)$ for all $v \in G$ and it suffices to repeat the arguments of the end of the proof of proposition 4.3.

In order to study the subdifferential of a composite functional $y^{\prime}$ of, let us recall the following notion. A linear mapping $A$ of an ordered vector space into another one is positive if $A x \geqslant 0$ for every $y \geqslant 0$.

We shall denote by $F_{+}^{\prime}$ the dual positive cone of $F^{\prime \prime}$, that is $F_{+}^{\prime}=\left\{y^{\prime} \in F^{\prime \prime} \mid y^{\prime}\left(F_{+}\right) \geqslant 0\right\}$.

4.5. LEMMA. - Let $G$ be a topological vector space which is an order complete vector lattice and let $A$ be a positive continuous linear mapping of $F$ into $G$. Suppose that $f$ is a mapping of $E$ into $F$ which is compactly lipschitzian at a point $\bar{x} \in E$. Then $A$ of is compactly lipschitzian at $\bar{x}$ and $(A \circ f)^{0}(\bar{x} ; v) \leqslant A\left(f^{\circ}(\bar{x} ; v)\right)$ for each $v \in E$ with the convention $A(+\infty)=+\infty$.

Proof. - By proposition 1.6 $A$ of is compactly lipsehitzian at $\vec{x}$. Choose now a mapping $K$ of $E$ into Comp $(F)$ and a mapping $r$ of $] 0,1] \times E \times E$ into $F$ verifying the conditions of definition 1.1. Let $v$ be any point in $E$ and let $l=\lim _{j \in J} q_{A \circ f}\left(t_{j}, x_{j} ; v\right)$ be any point in $D_{A \text { of }}(\bar{x} ; v)$. There exists $j_{0} \in J$ such that $q_{f}\left(t_{j}, x_{j} ; v\right)-r\left(t_{j}, x_{j} ; v\right) \in K(v)$ for each $j \geqslant j_{0}$. Since $K(v)$ is compact in $F$, there is a convergent subnet $\left(q_{f}\left(t_{\alpha(i)}, x_{\alpha(i)} ; v\right)\right)_{i \in I}$. Then using the continuity of the mapping $A$, we obtain

$$
l=\lim _{i \in I} q_{A \text { of }}\left(t_{\alpha(i)}, x_{\alpha(i)} ; v\right)=A\left(\lim _{i \in I} q_{f}\left(t_{\alpha(i)}, x_{\alpha(i)} ; v\right)\right) .
$$

Therefore, $l \in A\left(D_{f}(\bar{x} ; v)\right)$ and hence $D_{A \circ f}(\bar{x} ; v) \subset A\left(D_{f}(\bar{x} ; v)\right)$.

Finally, it follows from the positivity of the linear mapping $A$ that $(A \circ f)^{0}(x ; v) \leqslant$ $\leqslant A\left(f^{0}(\bar{x} ; v)\right)$.

REMARKS.

1) Since the inclusion $A\left(D_{f}(\bar{x} ; v)\right) \subset D_{A \text { of }}(\vec{x} ; v)$ is obvious, we have $A\left(D_{f}(\vec{x} ; v)\right)=$ $=D_{A \circ f}(\bar{x} ; v)$ for every $v \in E$.

2) Assume that $F$ is a normed vector space, that $g$ is a mapping of $F$ into $G$ which is strictly differentiable at $f(\bar{x})$ and that $\nabla g(f(\bar{x}))$ is a positive linear mapping 
of $F$ into $G$. If the mapping $f$ is continuous at $\bar{x}$, then $g \circ f$ is compactly lipsehitzian at $\bar{x}$ and we have $(g \circ f)^{\circ}(\bar{x} ; z) \leqslant \nabla g(f(\bar{x}))\left(f^{0}(\bar{x} ; z)\right)$ for every $z \in E$. Indeed, let $v$ be a point in $E$. On the one hand, consider a neighborhood $Q$ of $\bar{x}$ in $E$ and a real number $\eta \in] 0,1]$ such that $q_{f}(t, x ; v) \in K_{f}(v)+r_{f}(t, x ; v)$ for all $\left.\left.(t, x) \in\right] 0, \eta\right] \times \Omega$ and that $\left.\left.r_{f}(] 0, \eta\right] \times \Omega ; v\right)$ is topologically bounded in $F$. Since

$$
g(z)-g(y)=\nabla g(f(\bar{x})) \cdot(z-y)+\|z-y\| \varepsilon(z, y)
$$

with $\lim _{v \rightarrow(z, y)}=0$, we have for every $\left.\left.(t, x) \in\right] 0, \eta\right] \times \Omega$

$\underset{\substack{x \rightarrow f(\bar{x}) \\ y \rightarrow f(\bar{x})}}{(x)}$

$$
\begin{aligned}
l & =t^{-1}[g(f(x+t v))-g(f(x))] \\
& =\nabla g(f(\bar{x})) \cdot t^{-1}[f(x+t v)-f(x)]+t^{-1}\|f(x+t v)-f(x)\| \varepsilon(f(x+t v), f(x)),
\end{aligned}
$$

and hence

$$
l \in \nabla g(f(\bar{x}))\left(K_{f}(v)\right)+\nabla g(f(\bar{x})) \cdot r_{f}(t, x ; v)+t^{-1}\|f(x+t v)-f(x)\| \varepsilon(f(x+t v), f(x)) .
$$

Moreover, $\lim _{\substack{t, 0 \\ x \rightarrow \bar{\alpha}}} t^{-1}\|f(x+t v)-f(x)\| \varepsilon(f(x+t v), f(x))=0$, for the set

$$
\left.\left.K_{f}(v)+r_{f}(] 0, \eta\right] \times \Omega ; v\right)
$$

is topologically bounded in $F$ and $\lim _{\substack{t \neq 0 \\ x \rightarrow \bar{x}}} \varepsilon(f(x+t v), f(x))=0$ ( $f$ is continuous at $\bar{x}$ ). Therefore, $f \circ g$ is compactly lipschitzian at $\bar{x}$.

On the other hand, to prove the inequality $(g \circ f)^{0}(\bar{x} ; v) \leqslant \nabla g(f(\bar{x}))\left(f^{0}(\bar{x} ; v)\right)$ it suffices to use arguments similar to the ones of the proof of the above proposition.

4.6. Propostrion. - Let $y^{\prime}$ be a positive continuous linear functional on $F\left(y^{\prime} \in \bar{H}_{+}^{\prime}\right)$ and let $\bar{x}$ be a point in $E$ which is regular for a mapping $f$ of $E$ into $F$. Assume that $F_{+}$ is normal, then:

i) $\partial\left(y^{\prime} \circ f\right)(\bar{x}) \subset \mathrm{el}_{E_{\sigma}^{\prime}}\left[y^{\prime} \circ \partial f(\bar{x})\right]$, where $y^{\prime} \circ \partial f(\bar{x})=\left\{y^{\prime} \circ T \mid T \in \partial f(\bar{x})\right\}$;

ii) if, in addition, the order intervals are compact in $F$, we have $\partial\left(y^{\prime} \circ f\right)(\bar{x}) \subset y^{\prime} \circ \partial f(\bar{x})$.

Proof. - The above lemma says that $\left(y^{\prime} \circ f\right)^{\circ}(\bar{x} ; v) \leqslant y^{\prime}\left(f^{0}(\bar{x} ; v)\right)$ for all $v \in E$, and hence it ensures that $\bar{x}$ is a regular point for the function $y^{\prime}$ of.

Then, by proposition 3.4, we have

$$
\max \left\{\left\langle x^{\prime}, v\right\rangle \mid x^{\prime} \in \partial\left(y^{\prime} \circ f\right)(\bar{x})\right\} \leqslant \max \left\{\left\langle y^{\prime} \circ T, v\right\rangle \mid T \in \partial f(\bar{x})\right\} \quad \text { for every } v \in E .
$$

Thus, the Hahn-Banach separation theorem implies that assertion (i) holds.

For (ii) it suffices to apply proposition 3.4 to conclude that $\partial f(\bar{x})$ is compact in $L_{s}(E, F)$, and hence that $y^{\prime} \circ \partial f(\bar{x})$ is compact in $E_{\sigma}^{\prime}$ since the mapping of $L_{s}(E, F)$ into $E_{\sigma}^{\prime}$ defined by $T \mapsto y^{\prime} \circ T$ is continuous. 


\section{REMARKS.}

1) In general, the above inclusion cannot be replaced by an equality. Indeed, consider, for instance, two real valued functions $f_{1}$ and $f_{2}$ defined on $\mathbb{R}$ which are Lipschitz at a point $\bar{x} \in \mathbb{R}$ and which verify the relation $\partial f_{1}(\bar{x})+\partial f_{2}(\bar{x}) \notin \partial\left(f_{1}+f_{2}\right)(\bar{x})$. Define a mapping $f$ of $\mathbb{R}$ into $\mathbb{R}^{2}$ by $f(x)=\left(f_{1}(x), f_{2}(x)\right)$ for every $x \in \mathbb{R}$ and a positive linear mapping $y^{\prime}$ of $\mathbb{R}^{2}$ into $\mathbb{R}$ by $y^{\prime}\left(x_{1}, x_{2}\right)=x_{1}+x_{2}$ for every $\left(x_{1}, x_{2}\right) \in \mathbb{R}^{2}$. The mapping $f$ is Lipsehitz at $\bar{x}$ and we see that

$$
y^{\prime}(\partial f(\bar{x}))=y^{\prime}\left(\partial f_{1}(\bar{x}) \times \partial f_{2}(\bar{x})\right)=\partial f_{1}(\bar{x})+\partial f_{2}(\bar{x}) \not \partial\left(f_{1}+f_{2}\right)(\bar{x})=\partial\left(y^{\prime} \circ f\right)(\bar{x}) .
$$

2) If the assumptions of remark 2) following proposition 4.5 are verified with $G=\mathbb{R}$ and if $f$ is regular at $\bar{x}$, then using arguments similar to the ones of the above proposition, one obtains

$$
\partial(g \circ f)(\bar{x}) \subset \mathrm{cl}_{\bar{E}_{\sigma}^{\prime}}[\nabla g(f(\bar{x})) \circ \partial f(\bar{x})] .
$$

The following proposition gives a formula about the subdifferential of a product.

4.7. Proposition. - Assume that $E$ is a normed vector space. Let $f$ be a mapping of $E$ into $\vec{F}$ which is continuous and compactly lipsehitzian at a point $\vec{x} \in E$ and let $k$ be a real valued function which is defined on $E$ and strictly differentiable at $\vec{x}$. Then the mapping hi defined by $(k f)(x)=k(x) f(x)$ for all $x \in E$ is compactly lipschitzian at $\bar{x}$ and the following formula holds:

$$
\partial(k f)(\bar{x})=k(\bar{x}) \cdot \partial f(\bar{x})+\nabla k(\bar{x}) \cdot f(\bar{x})
$$

where

$$
k(\bar{x}) \cdot \partial f(\bar{x})=\{k(\bar{x}) T \mid T \in \partial f(\bar{x})\}
$$

and $\nabla k(\bar{x}) \cdot f(\bar{x})$ is the mapping of $E$ into $F$ defined by $v \mapsto \nabla k(\bar{x})(v) \cdot f(\bar{x})$.

Proof. - Let $v$ be any point in $E$. Consider a neighborhood $\Omega$ of $\vec{x}$ and a real number $\eta \in] 0,1]$ such that

$$
q_{f}(t, x ; v) \in K_{f}(v)+r_{f}(t, x ; v)
$$

and

$$
\left.\left.q_{k}(t, x ; v)=\nabla k(\bar{x})(v)+r_{k}(t, x ; v) \quad \text { for all }(t, x) \in\right] 0, \eta\right] \times \Omega
$$

Then, for every $(t, x) \in] 0, \eta] \times \Omega$, if we write

$$
\begin{aligned}
z=t^{-1}[k(x+t v) f(x+t v)-k(x) f(x)] & \\
& =k(x+t v) \cdot t^{-1}[f(x+t v)-f(x)]+t^{-1}[k(x+t v)-k(x)] \cdot f(x),
\end{aligned}
$$


we obtain

$$
z \in k(\vec{x}) K_{f}(v)+\nabla k(\bar{x})(v) \cdot f(\vec{x})+k(x+t v) \cdot r_{f}(t, x ; v)+r_{k}(t, x ; v) f(x)+d(t, x ; v)
$$

with $d(t, x ; v) \in(k(x+t v)-k(\bar{x})) K_{f}(v)+\nabla k(\bar{x})(v) \cdot(f(x)-f(\bar{x}))$. Since $\quad k$ and $f$ are continuous at $\vec{x}$ and that $K_{f}(v)$ is topologically bounded in $F$, it follows that $\lim _{t \psi_{0}} d(t, x ; v)=0$ and hence that the mapping $k f$ is compactly lipschitzian at $\vec{x}$. Now, let us prove that the formula of the proposition holds. By proposition 3.6 we may suppose that $k(\vec{x}) \geqslant 0$.

Let $\lim _{j \in J} q_{k f}\left(t_{j}, x_{j} ; v\right)$ be a point in $D_{k f}(\bar{x} ; v)$. There exists a subnet $\left(q_{k f}\left(t_{\alpha(i)}, x_{\alpha(i)} ; v\right)\right)_{i \in I}$ such that the subnets $\left(q_{k}\left(t_{\alpha(i)}, x_{\alpha(i)} ; v\right)\right)_{i \in I}$ and $\left(q_{f}\left(t_{\alpha(i)}, x_{\alpha(i)} ; v\right)\right)_{i \in I}$ are convergent. Then we have

$$
\lim _{j \in J} q_{k f}\left(t_{j}, x_{j} ; v\right)=k(\bar{x}) \lim _{i \in I} q_{f}\left(t_{\alpha(i)}, x_{\alpha(i)} ; v\right)+\left(\lim _{i \in I} q_{k}\left(t_{\alpha i(}, x_{\alpha(i)} ; v\right)\right) \cdot f(\bar{x})
$$

and hence $D_{k f}(\bar{x} ; v) \subset k(\bar{x}) \cdot D_{f}(\bar{x} ; v)+\nabla k(\bar{x})(v) \cdot f(\bar{x})$. Now, consider a point $\lim _{a \in A} q_{f}\left(t_{a}\right.$, $\left.x_{a} ; v\right) \in D_{f}(\bar{x} ; v)$. Since the net $\left(q_{k}\left(t_{a}, x_{a} ; v\right)\right)_{a \in A}$ is convergent, it follows that the net $\left(q_{k f}\left(t_{a}, x_{\alpha} ; v\right)\right)_{a \in A}$ is also convergent and that

$$
k(\bar{x}) \lim _{a \in A} q_{f}\left(t_{a}, x_{a} ; v\right)+\nabla k(\bar{x})(v) \cdot f(\bar{x})=\lim _{a \in A} q_{k f}\left(t_{a}, x_{a} ; v\right)
$$

and hence $k(\bar{x}) \cdot D_{f}(\bar{x} ; v)+\nabla k(\bar{x})(v) \cdot f(\bar{x}) \subset D_{k f}(\bar{x} ; v)$. Therefore, we have $(k f)^{0}(\bar{x} ; v)=$ $=k(\bar{x}) \cdot f^{0}(\bar{x} ; v)+\nabla k(\bar{x})(v) \cdot f(\bar{x})$ and the formula of the proposition is a consequence of the definition of the subdifferential.

REMARK. - If $F=\mathbb{R}$ and if $k$ is a real-valued function which is continuous, compactly lipsehitzian and regular at $\bar{x}$, then using arguments similar to the ones of the proof of the above proposition one can show that the function $k f$ is compactly lipschitzian at $\bar{x}$ and that

$$
\partial(k f)(\bar{x}) \subset k(\bar{x}) \partial f(\bar{x})+f(\bar{x}) \partial k(\bar{x})
$$

We turn now our attention to an important application of proposition 4.6. Let $\Sigma$ be a tribe on a set $S, \mu$ a positive measure on $\Sigma, E$ a separable Banach space, $\bar{x}$ a point in $E$ and $f$ a real valued function defined on $T \times E$ verifying the following conditions:

i) there exist a neighborhood $V$ of $\vec{x}$ in $E$ and a nonnegative function $\alpha \in \mathfrak{Q}^{1}(S, \mu)$ such that

$$
|f(s, x)-f(s, y)| \leqslant \alpha(s)\|x-y\| \quad \text { for all } x, y \in V ;
$$

ii) for each $x \in E$, the function $f(\cdot, x)$ is $\mu$-integrable. 
We may define a real function $g$ on $E$ by $g(x)=\int_{S} f(s, x) d \mu(s)$ and it is easily seen that $g$ is Lipschitz at $\vec{x}$. Using the theory of integrable multivalued mappings it has been proved in [41] that for each $\theta \in \partial g(\bar{x})$ there exists a weakly integrable mapping $\sigma$ of $S$ into $E^{\prime}$ such that $\theta=\int_{S} \sigma(s) d \mu(s)$ and $\sigma(s) \in \partial f_{s}(\bar{x}) \mu$-almost everywhere. Here, with the help of the above proposition we shall give a direct proof of this result.

Let us consider the mappings $k$ of $E$ into $L^{1}(\mathcal{S}, \mu)$ defined by $k(x)=[f(\cdot, x)]$ for all $x \in E$ and $y^{\prime}$ of $L^{1}(S, \mu)$ into $\mathbb{R}$ defined by $y^{\prime}([h])=\int h d \mu$ for all $[h] \in L^{1}(S, \mu)$ where the symbol $[\cdot]$ denotes the equivalence class for the almost-everywhere equality. We note that $y^{\prime}$ is a continuous positive linear functional, that the mapping $k$ is order Lipschitz at $\vec{x}$, and that by proposition 2.6 , if $L^{1}(S, \mu)$ is endowed with the weak topology, the point $\vec{x}$ is regular for the mapping $k$. Then proposition 4.6 ensures that there is an element $T \in \partial k(\bar{x})$ such that $\theta=y^{\prime} \circ T$. Also, we shall study the form of elements belonging to $\partial x(\bar{x})$.

Let us begin by proving a lemma which will be used in the next proposition.

4.8. LEMMA. - Let $v$ be a point in $E$. If we denote by $h$ the mapping of $S$ into $\mathbb{R}$ defined by $s \mapsto f_{s}^{0}(\bar{x} ; v)$, then $h$ is $\mu$-integrable and the equivalence class [h] of $h$ verifies the relation $k^{0}(\bar{x} ; v) \leqslant[h]$ in $L^{1}(S, \mu)$.

Proor. - The $\mu$-integrability of $h$ follows from (1). Now, since the mapping $(x, t) \mapsto x+t v$ is continuous there exists a positive real number $\varepsilon$ and a neighborhood $\Omega$ of $\bar{x}$ such that $\Omega+] 0, \varepsilon] v \subset V$. Let $\left(\Omega_{n}\right)_{n \in \mathbb{N}}$ be a countable neighborhood basis of $\bar{x}$ with $\Omega_{n+1} \subset \Omega_{n} \subset \Omega$ for each $n$ and $\left(\varepsilon_{n}\right)_{n}$ a decreasing sequence of real numbers in ] $0, \varepsilon]$ converging to zero. We can consider for each integer $n$ the $\mu$-measurable realvalued function $h_{n}$ defined on $S$ by $h_{n}(s)=\sup _{\substack{t \in\left[0, e_{n}\right] \\ x \in \Omega_{n}}} q_{f_{s}}(t, x ; v)$.

From relation (1) we have $h_{n} \in \mathcal{Q}^{1}(S, \mu)$ for each $n$. Then it follows from the relation $\sup _{k} q_{k}(t, x ; v) \leqslant\left[h_{n}\right]$ for each $n$ and relation (1) that

$$
\inf _{\substack{t \in\left[0, \varepsilon_{n}\right] \\ x \in \Omega_{n}}} \sup _{\substack{t \in j \in, \varepsilon_{n} \mathrm{~L} \\ x \in \Omega_{n}}} q_{\Omega_{i}}(t, x ; v) \leqslant \inf _{n}\left[h_{n}\right]=\lim _{n}\left[h_{n}\right]=\left[\lim _{n} h_{n}\right],
$$

where $\lim _{n}\left[h_{n}\right]$ denotes the limit of the sequence $\left(\left[h_{n}\right]\right)_{n}$ in $L^{1}(S, \mu)$ equipped with the strong topology. Finally, since the definition of $k^{0}(\bar{x} ; v)$ ensures that $k^{0}(\bar{x} ; v) \leqslant$ $\leqslant \inf \sup q_{k}(t, x ; v)$, we may conelude that $k^{0}(\bar{x} ; v) \leqslant[h]$.

n $\left.t \in \underset{x \in \Omega_{n}}{ }\right]$

The assertion will be proved if we construct a family $(\sigma(s))_{s \in S}$ of linear mappings of $E$ into $\mathbb{R}$ such that the mapping $\sigma(\cdot) \cdot v \in T(v)$ for each $v \in E$ (note that $T(v) \in$ $\left.\in L^{1}(S, \mu)\right)$ and that for $\mu$-almost every $s \in S, \sigma(s) \cdot v \leqslant f_{s}^{0}(\bar{x} ; v)$ for all $v \in E$.

This result is established in the following.

4.9. Proposition. - Under the above assumptions there exists a family $(\sigma(s))_{s \in S}$ of linear mappings of $E$ into $\mathbb{R}$ verifying the preceding relations. 
Proof. - Let $\left(e_{m}\right)_{m \in \mathbb{N}}$ be a total free sequence in $E$. For each integer $m$ choose a $\mu$-integrable real-valued function $h_{m} \in T\left(e_{m}\right)$. Consider the vector subspace $G$ generated by the family $\left(e_{m}\right)_{m \in \mathbb{N}}$ and for each $s \in S$ define a linear mapping $\varrho(s)$ of $G$ into $\mathbb{R}$ by $\varrho(s) \cdot\left(\sum_{m \leqslant p} \lambda_{m} e_{m}\right)=\sum_{m \leqslant p} \lambda_{m} h_{m}(s)$ for all $\lambda_{m} \in \mathbb{R}$ and $p \in \mathbb{N}$. Since $T$ is linear, it follows that the function $\varrho(\cdot) \cdot z \in T(z)$ for each $z \in G$. By the above lemma we have for each element $\sum_{m \leqslant p} \gamma_{m} e_{m}$ with $\gamma_{m} \in Q$ (the rational number system) $\varrho(s)\left(\underset{m \leqslant p}{\sum} \gamma_{m} e_{m}\right) \leqslant$ $\leqslant f_{s}^{0}\left(\bar{x} ; \sum_{m \leqslant p} \gamma_{m} e_{m}\right)$ for $\mu$-almost every $s \in S$. Hence there exists a $\mu$-negligible subset $S_{0}$ of $S$ such that for each $s \notin s_{0}, \varrho(s) \cdot\left(\sum_{m \leqslant p} \gamma_{m} e_{m}\right) \leqslant f_{s}^{0}\left(\bar{x} ; \sum_{m \leqslant p} \gamma_{m} e_{m}\right)$ for all $\sum_{m \leqslant p} \gamma_{m} e_{m}$ with $\gamma_{m} \in \mathbb{Q}$ and $p \in \mathbb{N}$. Since the mapping $f_{s}^{0}(\bar{x} ; \cdot)$ is continuous on $E$ for each $s \in S$, we obtain that for each $s \notin S_{0}, \varrho(s) \cdot z \leqslant f_{s}^{0}(\bar{x} ; z)$ for all $z \in G$. Hence for each $s \notin S_{0}$ the linear functional $\varrho(s)$ is continuous on $G$ for the topology induced by the topology of $E$. Therefore, for each $s \notin S_{0}$ we can extend $\varrho(s)$ to a continuous linear functional $\sigma(s)$ defined on $E$ and it is obvious that $\sigma(s) \cdot v \leqslant f_{s}^{0}(\bar{x} ; v)$ for all $v \in E$. If $s \in S_{0}$, we choose an element $\sigma(s) \in E^{\prime}$. It remains to show that $\sigma(\cdot) \cdot v \in T(v)$ for each $v \in E$.

Write $v=\lim _{n} z_{n}$ with $z_{n} \in G$ for each $n$. By relation (1) we have $[\sigma(\cdot) \cdot v]=$ $=\lim _{n}\left[\sigma(\cdot) z_{n}\right]$. Since $T$ is continuous, we may conclude that $[\sigma(\cdot) \cdot v]=T(v)$.

We are going to consider now the subdifferential of a pointwise supremum. For this study we shall need the following notions.

4.10. DEFInI'ION, - A topological vector lattice is an ordered topological vector space which is a vector lattice and which has a neighborhood basis $\{V\}_{V}$ of the origin such that $V=\bigcup_{x \in V}\{y|| y|\leqslant| x \mid\}$, where the symbol $|\cdot|$ denotes the absolute value. It is shown in [32] that an ordered topological vector space which is a vector lattice is a topological vector lattice if and only if the positive cone is normal and the lattice operations are continuous.

In proposition 4.13 we shall make use of the following result of KUTATELADZE in $[19]$.

4.11. Proposition. - Let $\left(g_{p}\right)_{1 \leqslant p \leqslant n}$ be $n$ sublinear mappings of a vector space $X$ into a complete vector lattice $Y$. If $g$ is the mapping of $X$ into $Y$ defined by $g(x)=$ $=\sup f_{p}(x)$ for every $x \in X$ and if $\partial_{a} g(0)$ denotes the algebraic convex subdifferential $1 \leqslant y \leqslant n$ of $g$ at the origin, then

$$
\partial_{a} g(0)=\bigcup\left\{\sum_{p=1}^{n} \partial_{a}\left(l_{p} \circ g_{p}\right)(0) \mid l_{p} \in L^{+}(Y, Y), \sum_{p=1}^{n} l_{p}=\mathbf{I} d_{Y}\right\},
$$

where $L_{+}(Y, Y)$ denotes the set of all positive linear mapping of $Y$ into itself and $\mathrm{Id}_{Y}$ the identity mapping on $Y$. 
Before giving our statement about the pointwise supremum let us prove the following two lemmas.

4.12. LeMñA. - Let $X$ be an order complete vector space and $l$ a linear mapping of $X$ into itself veritying $0 \leqslant l \leqslant \mathrm{Id}_{X}$, that is $0 \leqslant l(x) \leqslant x$ for all $x$ in the positive cone $X_{+}$ of $X$.

1) If $A$ is a subset of $X$ which is bounded above, then we have $\sup l(A)=l(\sup A)$.

2) If, in addition, $X$ is a topological vector lattice, then $l$ is continuous.

\section{Proof.}

1) Since $l$ is positive, we have $l(x) \leqslant l(\sup A)$ for all $x \in A$ and hence $l(A)$ is bounded above. Moreover for each $x \in A$ we have

$$
0 \leqslant l(\sup A)-l(x)=l(-x+\sup A) \leqslant-x+\sup A,
$$

for $l \leqslant \operatorname{Id}_{X}$ Therefore, we obtain that $0=\inf _{x \in A}(-x+\sup A)=\inf _{x \in A}(l(\sup A)-l(x))$ and hence $\sup _{x \in A} l(x)=l(\sup A)$.

2) Suppose now that $X$ is a topological vector lattice and consider any net $\left(x_{j}\right)_{j \in J}$ in $X$ converging to zero. By what precedes it is easily seen that $|l(x)|=$ $=l(|x|) \leqslant|x|$ for all $x \in X$. Also we have

$$
-\left|x_{j}\right| \leqslant l\left(x_{j}\right) \leqslant\left|x_{j}\right|
$$

for each $j \in J$ and hence $\lim _{j \in J} l\left(x_{j}\right)=0$.

4.13. LemMa. - Assume that $F$ is a topological vector lattice. Let $l$ be a linear mapping of $F$ into itself verifying $0 \leqslant l \leqslant \operatorname{Id}_{F}$ and let $f$ be a mapping of $E$ into $F$ which is compactly lipschitzian and regular at a point $\bar{x} \in E$. The mapping lof is compactly lipsehitzian and regular at $\vec{x}$ and $(l \circ f)^{\circ}(\vec{x} ; v)=l\left(f^{0}(\bar{x} ; v)\right)$ for every $v \in E$.

Proof. - It follows from lemma 4.12 and proposition 1.2 that the mapping $l$ of is compactly lipschitzian at $\bar{x}$. Let $v$ be any point in $E$. Since $f$ is regular at $\bar{x}$, the set $D_{f}(\bar{x} ; v)$ is bounded above. Moreover, by remark 1 ) following lemma 4.5 we have $l\left(D_{f}(\bar{x} ; v)\right)=D_{l \circ f}(\bar{x} ; v)$. Therefore, according to lemma 4.12 we may conclude that $(l \circ f)^{\circ}(\vec{x} ; v)=l\left(f^{\circ}(\bar{x} ; v)\right)$.

4.14. Proposition. - Let $\left(f_{p}\right)_{1 \leqslant p \leqslant n}$ be $n$ mappings of $E$ into $F$ which are compactly lipschitzian and regular at a point $\vec{x}$ and let $f$ be the mapping of $E$ into $F$ defined by $f(x)=\sup _{1 \leqslant p \leqslant n} f_{p}(x)$. Assume that $F$ is a topological vector lattice and that the mapping $f$ 
is compactly lipschitzian at $\bar{x}$. Then $f$ is regular at $\bar{x}$ and the following holds:

$$
\partial f(\vec{x}) \subset \bigcup\left\{\sum_{p=1}^{n} \partial\left(l_{p} \circ f_{p}\right)(\bar{x}) \mid l_{p} \in L_{+}(F, F), \sum_{p=1}^{n} l_{p}=\operatorname{Id}_{F}\right\} .
$$

Proof. - Let us consider the continuous mapping of $F^{n}$ into $F$ defined by $h\left(x_{1}, \ldots, x_{n}\right)=\sup \left(x_{1}, \ldots, x_{n}\right)$. If we prove that $f^{0}(\bar{x} ; y) \leqslant \sup f_{p}^{0}(\bar{x} ; y)$ for all $y \in E, f$ will be regular at $\bar{x}$ because of the continuity of $h$ and the normality of $F_{+}$and the result will be a consequence of Kutateladze's proposition, lemma 4.1, proposition 3.7 and lemma 4.13 .

For each $p, 1 \leqslant p \leqslant n$, let us choose a mapping $K_{y}$ of $E$ into $\operatorname{Comp}(F)$ and a mapping $r_{p}$ of $\left.] 0,1\right] \times E \times E$ into $F$ verifying definition 1.1 for the mapping $f_{p}$ and the point $\bar{x}$.

Let $v$ be any point in $E$ and $\lim _{j \in J} q_{f}\left(t_{j}, x_{j} ; v\right)$ any point in $D_{f}(\bar{x} ; v)$. There exists $j_{0} \in J$ such that for each $j \geqslant j_{0}$ and each $p, 1 \leqslant p \leqslant n, q_{f_{p}}\left(t_{j}, x_{j} ; v\right)-r_{p}\left(t_{j}, x_{j} ; v\right) \in K_{p}(v)$.

Thus, it follows from the compactness of the subsets $K_{p}(v)$ and the relations $\lim _{i \downarrow 0} r_{p}(t, x ; v)=0$ that there are $p$ subnets $\left(q_{f_{p}}\left(t_{\alpha(i)}, x_{\alpha(i)} ; v\right)\right)_{i \in I}$ which are convergent. $x \rightarrow x$

If we note that we have

$$
t^{-1}[f(x+t v)-f(x)] \leqslant \sup _{p} t^{-1}\left[f_{p}(x+t v)-f_{p}(x)\right]
$$

for each positive number $t$ and each $x \in E$, then using the continuity of $h$ we may conclude that

$$
\begin{aligned}
\lim _{j \in J} q_{f}\left(t_{j}, x_{j} ; v\right) & =\lim _{i \in I} q_{j}\left(t_{\alpha(i)}, x_{\alpha(i)} ; v\right) \\
& \leqslant \lim _{i \in I}\left(\sup _{p} q_{f_{p}}\left(t_{\alpha(i)}, x_{\alpha(i)} ; v\right)\right) \\
& =\sup _{p}\left(\lim _{i \in I} q_{f_{p}}\left(t_{\alpha(i)}, x_{\alpha(i)} ; v\right)\right) \\
& \leqslant \sup _{p} f_{p}^{0}(\bar{x} ; v) . \quad \square
\end{aligned}
$$

REMARK. - If the mappings $f_{p}$ are order Lipsehitz at $\bar{x}$, then $f$ is also order Lipschitz at $\bar{x}$. Indeed, let $v$ be a point in $E$. There exist a neighborhood $\Omega$ of $\bar{x}$ in $E$ and a real number $\eta \in] 0,1]$ such that, for each $p$ and each $(t, x) \in] 0, \eta] \times \Omega, t^{-1} f_{p}(x+t v) \in$ $\in t^{-1} f_{p}(x)+\left[\underline{h}_{p}(v), \bar{h}_{p}(v)\right]+r_{p}(t, x ; v)$ where the mappings $\underline{h}_{p}, \bar{h}_{p}$ and $r_{p}$ verify the conditions of definition 1.5. If we put $\underline{h}(v)=\inf _{p} \underline{h}_{p}(v), \bar{h}(v)=\sup _{p} \bar{h}_{p}(v), \bar{r}(t, x ; v)=$ $=\sup _{p} r_{p}(t, x ; v)$ and $\underline{r}(t, x ; v)=\inf r_{p}(t, x ; v)$, it is an easy matter to verify that $q_{f}(t, x ; v) \in[\underline{h}(v), \bar{h}(v)]+[\underline{r}(t, x ; v), \bar{r}(t, x ; v)]$. Therefore, there exists

$$
r(t, x ; v) \in[\underline{r}(t, x ; v), \bar{r}(t, x ; v)]
$$

such, that $q_{f}(t, x ; v) \in[\underline{h}(v), \bar{h}(v)]+r(t, x ; v)$ for each $\left.\left.(t, x) \in\right] 0, \eta\right] \times \Omega$. Thus, $f$ is order Lipschitz at $\bar{x}$. 


\section{5. - Subdifferential of a continuous vector sum.}

In this section $F$ will be a separable Banach lattice, that is a Banach lattice which is topologically separable, and $F$ will be order complete. Thus, there is a norm $\|\cdot\|$ on $F$ defining the topology of $F$ and verifying the following: $\|x\| \leqslant\|y\|$ if $|x| \leqslant|y|$ (see [32]). $F$ will be endowed with such a norm. We shall assume that $F$ has the Radon-Nikodym property, that is for every positive measure space $(L, \Lambda, \lambda)$ and for every countably additive set function $m: A \rightarrow F$ which satisfies

$$
\sup \left\{\sum_{k=0}^{n}\left\|m\left(A_{k}\right)\right\| \mid n \in \mathbb{N}, A_{0}, \ldots, A_{n} \in A \text { pairwise disjoint }\right\}<\infty
$$

and which is absolutely continuous with respect to $\lambda$ there exists $h \in \mathfrak{R}_{F}^{1}(L, \lambda)$ verifying $m(A)=\int_{A} h d \lambda$ for all $A \in \Lambda$.

We shall also assume that each order interval of $F$ is weakly compact. Then by theorem 5.1 in KAWAI [17] every increasing net in $F$ which is bounded above converges to its least upper bound for the strong topology. This can be also seen by using propositions 3.1 and 3.4 in Peressini [32].

REMARK. - These assumptions are verified by the reflexive separable Banach lattices and by the space $l^{1}$ of all real sequences $\left(z_{n}\right)_{n \in \mathbb{N}}$ such that $\sum_{n \in \mathbb{N}}\left|z_{n}\right|<\infty$ endowed with the norm $\|z\|=\sum_{n \in \mathbb{N}}\left|\tilde{z}_{n}\right|$ and with its natural order structure.

In the following $(S, \Sigma, \mu)$ will denote a complete measured space. $B y \mathfrak{L}_{F}^{1}(S, \mu)$ we shall mean the space of Bochner $\mu$-integrable mappings of $S$ into $F$ and by $F_{\sigma}$ the space $F^{\prime}$ endowed with the $\sigma\left(F^{\prime}, F^{\prime}\right)$-topology. We shall assume that $E$ is a separable Banach space and that $f$ is a mapping of $S \times E$ into $F$ verifying the following conditions for a point $\bar{x} \in E$ and a neighborhood $\Omega$ of $\bar{x}$ in $E$ :

i) there exist two mappings $k_{1}$ and $k_{2}$ of $S \times E$ into $F$ with $k_{1}(\cdot, x)$ and $k_{2}(\cdot, x)$ belonging to $\mathfrak{\Omega}_{F}^{1}(S, \mu)$ for each $x \in \Omega$, a mapping $\varepsilon$ of $\left.\left.S \times\right] 0,1\right] \times E \times E$ into $F$ with $\lim _{\substack{t \downarrow 0 \\ x \rightarrow \bar{x}}} \varepsilon(s, t, x ; v)=0$ for each $(s, v) \in S \times E$ and $\varepsilon(\cdot, t, x ; v) \in \mathbb{R}_{\mathrm{F}}^{1}(S, \mu)$ for each $(t, x, v) \in] 0,1] \times \Omega \times E$, a mapping $\beta$ of $S \times E$ into $\mathbb{R}$ with $\beta(\cdot, v) \in$ $\in \mathfrak{R}_{\mathbb{R}}^{1}(S, \mu)$ for each $v \in E$, and a nonnegative real-valued function $\alpha \in \mathfrak{R}_{\mathbb{R}}^{1}(S, \mu)$ such that:

a) $t^{-1}(f(s, x+t v)-f(s, x)) \in\left[k_{1}(s, v), k_{2}(s, v)\right]+\varepsilon(s, t, x ; v)$ for each $(s, t, x, v) \in$ $\in S \times] 0,1] \times \Omega \times E$ and

b) $\left\|k_{i}(s, v)\right\| \leqslant \alpha(s)\|v\|$ for each $(s, v) \in S \times E$ and $i=1,2$ and $\|\varepsilon(s, t, x ; v)\| \leqslant$ $\leqslant \beta(s, v)$ for each $(s, t, x, v) \in S \times] 0,1] \times \Omega \times E$;

ii) for each $x \in E$, the mapping $f(\cdot, x)$ is Bochner $\mu$-integrable;

iii) the mapping $f(s, \cdot)$ of $E$ into $F_{\sigma}$ is continuous on $\Omega$ for each $s \in S$. 
We may define a mapping $g$ of $E$ into $F$ by $g(x)=\int_{S} f(s, x) d \mu(s)$ and using lemma 5.1 and the above assumptions it is easily seen that $g$ is compactly lipschitzian at $\bar{x}$ as a mapping of $E$ into $F_{\sigma}$. We are interested in the subdifferential of $g$ at the point $\bar{x}$.

We shall begin with the following lemma.

5.1. LEMrMa. - If $h$ is a positive mapping in $\mathfrak{Q}_{F}^{\mathbf{1}}(S, \mu)$, then $\int_{S} h(s) d \mu(s) \geqslant 0$ in $F$.

Proof. - Since $F_{+}$is a closed convex cone in $F$, the Hahn-Banach theorem says that it suffices to show that $\left\langle y^{\prime}, \int_{S} h(s) d \mu(s)\right\rangle \geqslant 0$ for every $y^{\prime} \in F_{+}^{\prime}$.

For such an element $y^{\prime}$ we have

$$
\left\langle y^{\prime}, \int_{S} h(s) d \mu(s)\right\rangle=\int_{S}\left\langle y^{\prime}, h(s)\right\rangle d \mu(s) \geqslant 0
$$

since $\mu$ is a positive measure.

In the sequel we shall need a proposition of NEUMANn [27].

5.2. Proposition. - Let $X$ be a separable Banach space and $Y$ be an ordercomplete Banach lattice. Assume that the space $Y$ has the Radon-Nikodym property. Let $p$ be a mapping of $S \times X$ into $Y$ such that

i) for each $s \in S, p(s, \cdot)$ is a sublinear mapping of $X$ into $Y$;

ii) for each $x \in X, p(\cdot, x)$ is Bochner $\mu$-measurable;

iii) there exists a nonnegative real-valued function $h \in \Omega_{\mathbb{B}}^{1}(S, \mu)$ verifying $\|p(s, x)\| \leqslant$ $\leqslant h(s)\|x\|$ for $a l l s \in \mathbb{S}$ and $x \in X$.

If $T \in L(X, Y)$ verifies $T(x) \leqslant \int_{S} p(s, x) d \mu(s)$, then there exists a family $\left(T_{s}\right)_{s \in S}$ of continuous linear mappings of $X$ into $Y$ such that:

a) the mapping $s \mapsto T_{s}(x)$ is Bochner $\mu$-integrable for each $x \in X$;

b) $T(x)=\int T_{s}(x) d \mu(s)$ for each $x \in X$;

c) for each $s \in S, T_{s}(x) \leqslant p(s, x)$ for all $x \in X$.

REMarK. - This proposition has been formulated by SATNT-PIERRE in [37, 38] for the separable dual Banach spaces and has been independently proved by NEUMANN in [27] for the Banach spaces which have the Radon-Nikodym property.

In order to apply Neumann's proposition we are going to show that the mapping $s \mapsto f_{s}^{0}(\bar{x} ; v)$ is Bochner $\mu$-integrable.

Since $F$ is a separable Banach space, its topological dual $F^{\prime \prime}$ is $\sigma\left(F^{\prime \prime}, F\right)$-separable. Let us fix a $\sigma\left(F^{\prime}, F^{\prime}\right)$-dense sequence $\left(e_{n}^{\prime}\right)_{n \in \mathbb{N}}$ in $F^{\prime}$ and let us consider the countable 
family of semi-norms $\left(p_{n}\right)_{n \mathrm{NE}}$ defined on $F$ by $p_{n}(x)=\left\langle e_{n}^{\prime}, x\right\rangle \mid$ for every $x \in F$. The locally convex topology on $F^{\prime}$ generated by the family $\left(p_{n}\right)_{n \in \mathbb{N}}$ will be called the $\theta$-topology and $F_{\theta}$ will mean the space $F$ endowed with the $\theta$-topology. It is easily seen that this topology is metrizable and coarser than the $\sigma\left(F, F^{\prime}\right)$-topology. Moreover, on every $\sigma\left(F, F^{\prime}\right)$-compact subset of $F^{\prime}$ these two topologies coincide.

5.3. LEMMA. - The multivalued function $s \mapsto \mathrm{cl}_{F_{\sigma}}\left[q_{f_{s}}(] 0, \varepsilon[\times W ; v)\right]$ from $S$ to $F_{\theta}$ is $\Sigma$-measurable for each $\varepsilon>0$ and each neighborhood $W$ of $\bar{x}$ verifying $W+] 0, \varepsilon[v \subset \Omega$ and $W \subset \Omega$.

Proof. - Let $v$ be a point in $E$ and let $\left(t_{n}, x_{n}\right)_{n}$ be a dense sequence in $] 0, \varepsilon[\times W$. Since $q_{f_{s}}(\cdot, \cdot ; v)$ is continuous on $] 0, \varepsilon\left[\times W\right.$ for the $\sigma\left(F, F^{\prime}\right)$-topology of $F$, the sequence $\left(q_{f_{s}}\left(t_{n}, x_{n} ; v\right)\right)$ is dense in $\operatorname{cl}_{F_{v}}\left[q_{f_{s}}(] 0, \varepsilon[\times W ; v)\right]$ which is $\sigma$-compact and hence 0 -compact. Noting that $s \mapsto q_{f_{s}}\left(t_{n}, x_{n} ; v\right)$ is $\Sigma$-measurable, the lemma follows from theorem III.9 of CASTAING-VALADIER [5].

5.4. Corollary. - The multivalued funotion $s \mapsto D_{f_{s}}(x ; v)$ from $S$ to $F_{\theta}$ is $\Sigma$-measurable for each $v \in \mathbb{E}$.

Proof. - Choose a real number $\varepsilon>0$ and a neighborhood $W$ of $\bar{x}$ verifying $W+] 0, \varepsilon\left[v \subset \Omega\right.$ and $W \subset \Omega$. It suffices to note that, if $\left(W_{n}\right)_{n \in \mathbb{N}}$ is a countable neighborhood basis of $\bar{x}$ with $W_{n} \subset W$ for each $n$ and if $\left(\varepsilon_{n}\right)_{n \in \mathbb{N}}$ is a decreasing sequence of $] 0, \varepsilon\left[\right.$ converging to zero, we have $D_{f_{s}}(\bar{x} ; v)=\bigcap_{n \in \mathbb{N}} \operatorname{cI}_{F_{\sigma}}\left(q_{f_{s}}(] 0, \varepsilon_{n}[\times W ; v)\right)$ with $\operatorname{cl}_{F_{\sigma}}(] 0, \varepsilon_{n}\left[\times W_{n} ; v\right) \sigma$-compact and hence $\theta$-compact and to apply proposition III.4 in CastaING-VALADIER [5].

In the proof of the next proposition we shall make use of the following result.

5.5. LeiMMa. - Let $X$ be an order-complete topological vector space. If a subset $D$ is dense in a nonvoid subset $R$ of $X$ which is bounded above, then we have $\sup D=\sup R$.

Proof. - The inequality $\sup D \leqslant \sup R$ is obvious. Let $r$ be any point in $R$. Since $D$ is dense in $R$, there exists a convergent net $\left(d_{j}\right)_{j \in J}$ in $D$ verifying $r=\lim _{j \in J} d_{j}$. For each $j \in J$ we have $d_{j} \leqslant \sup D$. Therefore, we may conclude that $r=\lim _{j \in J} d_{j} \leqslant$ $\leqslant \sup D$ since the positive cone $X_{+}$is closed.

REMARK. - This lemma has the following consequence. Let $R$ be a subset of an order-complete locally convex space $X$. If $R$ is bounded above, then we have $\sup R=\sup (\overline{c o} R)$, where the symbol $\overline{c 0}$ denotes the closed convex hull operation with respect to a topology compatible with the duality between $X$ and its topological dual $X^{\prime}$. Indeed it is easily seen that $\sup R=\sup (\operatorname{co} R)$ and hence we may apply the preceding result. 
5.6. Proposition. - The mapping $s \mapsto f_{s}^{0}(\bar{x} ; v)$ is Bochner-measurable for each $v \in E$.

Proof. - Let $v$ be any fixed point in $E$. Since the multifunction $s \mapsto D_{f_{s}}(\bar{x} ; v)$ from $S$ to $F_{\theta}$ is measurable with nonempty compact values, there exists a sequence $\left(h_{n}\right)_{n \in \mathbb{N}}$ of measurable mappings of $S$ into $F_{\theta}$ verifying $D_{f_{s}}(\bar{x} ; v)=\operatorname{cl}_{F_{\theta}}\left(\left\{h_{n}(s) \mid n\right\}\right)=$ $=\mathrm{cI}_{F_{\sigma}}\left(\left\{h_{n}(s) \mid n\right\}\right)$ for all $s \in S$. Moreover, from the $\sigma\left(F^{\prime}, F\right)$-density of the family $\left(e_{n}^{\prime}\right)_{n \in \mathbb{N}}$ it follows that the real-valued function $s \mapsto\left\langle x^{\prime}, h_{m}(s)\right\rangle$ is measurable for each $x^{\prime} \in F^{\prime \prime}$ and each $m \in \mathbb{N}$. Therefore the mappings $h_{n}$ are Bochner measurable since the space $F$ is strongly separable. Now denote by $G=\left\{g_{m} \mid m \in \mathbb{N}\right\}$ the set of all convex combinations with rational coefficients of the mappings $h_{n}$. For each $s \in S$ the subset $G_{s}=\left\{g_{m}(s) \mid m \in \mathbb{N}\right\}$ is strongly dense in $\overline{c 0}\left[D_{f_{s}}(\bar{x} ; v)\right]$. Thus lemma 5.5 and the remark which follows it imply that

$$
\begin{aligned}
f_{s}^{0}(\bar{x} ; v) & =\sup \overline{c o}\left[D_{f_{s}}(\bar{x} ; v)\right] \\
& =\sup \left\{g_{m}(s) \mid m \in \mathbb{N}\right\} \\
& =\sup _{n}\left(\sup \left\{g_{m}(s) \mid m \leqslant n\right\}\right) \\
& =\lim _{n \rightarrow \infty}\left(\sup \left\{g_{m}(s) \mid m \leqslant n\right\}\right),
\end{aligned}
$$

which completes the proof since the lattice operations are strongly continuous.

We can now give a proposition which will enable us to state our result about the subdifferential of $g$ at the point $\bar{x}$.

5.7. Proposition. - The vector valued function $s \mapsto f_{s}^{0}(\bar{x} ; v)$ is Bochner $\mu$-integrable and the inequality $g^{0}(\bar{x} ; v) \leqslant \int_{S} f_{s}^{0}(\bar{x} ; v) d \mu(s)$ holds for every $v \in E$.

Proof. - Let $v$ be any fixed point in $E$. Put $k=\sup \left(\left|k_{1}\right|,\left|k_{2}\right|\right)$. Since $\alpha \in \mathfrak{Q}_{\mathbb{R}}^{1}(S, \mu)$, the $\mu$-integrability of the above mapping is a direct consequence of proposition $\mathbf{5 . 6}$ and the inequalities

$$
\left\|f_{s}^{0}(\bar{x} ; v)\right\| \leqslant\|k(s, v)\| \leqslant 2 \alpha(s)\|v\|
$$

Now consider any point $z \in D_{g}(\bar{x} ; v)$. Since the topology induced on $D_{g}(\bar{x} ; v)$ by the weak topology is metrizable, there exist a sequence $\left(t_{n}\right)_{n \in \mathbb{N}}$ of positive numbers converging to zero and a sequence $\left(x_{n}\right)_{n}$ in $\Omega$ converging to $\bar{x}$ such that we have $x_{n}+t_{n} v \in \Omega$ for all $n$ and $z=\lim _{n \rightarrow \infty} q_{g}\left(t_{n}, x_{n} ; v\right)$.

If we show that the relation $\left\langle y^{\prime}, z\right\rangle \leqslant\left\langle y^{\prime}, \int_{S} f_{s}^{0}(\bar{x} ; v) d \mu(s)\right\rangle$ holds for every $y^{\prime} \in F_{+}^{1}$, then according to the Hahn-Banach theorem the assertion will be proved. Indeed. 
for such an element $y^{\prime}$ it follows from lemma 4.5 and condition (i) that

$$
\begin{aligned}
\left\langle y^{\prime}, z\right\rangle & =\lim _{n \rightarrow \infty}\left\langle y^{\prime}, t_{n}^{-1} \int_{S}\left[f_{s}\left(x_{n}+t_{n} v\right)-f_{s}\left(x_{n}\right)\right] d \mu(s)\right\rangle \\
& =\lim _{n \rightarrow \infty} \int_{S} t_{n}^{-1}\left[\left(y^{\prime} \circ f_{s}\right)\left(x_{n}+t_{n} v\right)-\left(y^{\prime} \circ f_{s}\right)\left(x_{n}\right)\right] d \mu(s) \\
& \leqslant \int_{S} \lim \sup ^{-1} t_{n}^{-1}\left[\left(y^{\prime} \circ f_{s}\right)\left(x_{n}+t_{n} v\right)-\left(y^{\prime} \circ f_{s}\right)\left(x_{n}\right)\right] d \mu(s) \\
& \leqslant \int_{S}\left(y^{\prime} \circ f_{s}\right)^{0}(\bar{x} ; v) d \mu(s) \\
& \leqslant \int_{S} y^{\prime}\left(f_{s}^{0}(\bar{x} ; v)\right) d \mu(s) \\
& =\left\langle y^{\prime}, \int_{S} f_{s}^{0}(\bar{x} ; v) d \mu(s)\right\rangle .
\end{aligned}
$$

5.9. ConollaRY. - Under the above assumptions for each $T \in \partial g(\bar{x})$, there exists a family $\left(T_{s}\right)_{s \in S}$ of continuous linear mappings of $E$ into $F$ such that

a) $T_{s} \in \partial f_{s}(\bar{x})$ for every $s \in S$;

b) the mapping $s \mapsto T_{s}(v)$ is Bochner $\mu$-integrable for every $v \in E$;

c) $T(v)=\int_{S} T_{s}(v) d \mu(s)$ for every $v \in \mathbb{E}$.

Proor. - Let $T$ be any fixed point in $\partial g(\bar{x})$. For each $v \in E$ the inequalities $T(v) \leqslant g^{0}(x ; v) \leqslant \int_{S} f_{s}^{0}(\bar{x} ; v) d \mu(s)$ hold. Moreover we know that, for $k=\sup \left(\left|k_{1}\right|,\left|k_{2}\right|\right)$, we have $\left\|f_{s}^{0}(\bar{x} ; v)\right\| \leqslant\|k(s, v)\| \leqslant 2 \alpha(s)\|v\|, \alpha \in \mathbb{R}_{\mathbb{R}}^{1}(S \mu)$, and that the mapping $s \rightarrow f_{s}^{0}(x ; v)$ is Bochner-measurable. Thus, it follows from Neumann's proposition that there exists a family $\left(T_{s}\right)_{s \in S}$ of linear continuous mappings verifying condition s $(a),(b)$ and $(c)$.

ReMark. - Since the dual of $L(E, F)$ is $E \otimes F^{\prime}$, it is easily seen that the mapping $s \mapsto T_{s}$ of $B$ into $L(E, F)$ is Pettis-integrable and that its Pettis integral is equal to $T$.

\section{REFERENCES}

[1] J. P. Aubrr, Miero-cours "Gradients généralisés de Clarke 》, Centre de Recherches Mathématiques, no. 703, Université de Montréal, 1977.

[2] A. AUSLENDER, Minimisation sans contraintes de fonctions localement lipschitziennes: Application à la programmation mi-convexe, mi-différentiable, C. R. Acad. Sci. Paris, 284 (1977) pp. 959-961. 
[3] M. S. BazaraA - J. J. Goode, Necessary optimality criteria in Mathematical programming in normed linear spaces, J. Optimization Theory Appl., 11 (1973), pp. 235-244.

[4] N. BourbakI, Tariétés Différentiables et Analytiques, Fascicule de résultats, Hermann, Paris, 1967.

[5] C. Castaing - M. Valadier, Convex Analysis and Measurable Multifunctions, Lectures Notes in Mathematics 580, Springer-Verlag, Berlin, 1977.

[6] F. H. Clarke, Generalized gradients and applications, Trans. Amer. Math. Soc., 205 (1975), pp. 247-262.

[7] F. H. CLARKE, A new approach to Lagrange multipliers, Math. of Operations Res., 2 (1976), pp. $165-174$.

[8] F. H. CLARKE, Generalized gradients of Lipschitz functionals, MRC Technical report, Univ. of Wisconsin, Madison, August 1976.

[9] F. H. ClaRKE, On the inverse function theorem, Pacific J. Math., 64 (1976), pp. 97-102.

[10] A. A. Goldsteir, Optimisation of Lipschitz continuous functions, Math. Progr., 13 (1977), pp. 14-22.

[11] H. H. HALKIN, Mathematical programming without differentiability, in "Proceedings of MRC Symposium on the calculus of variations and optimal control, Sept. 1975 " (D. L. Russell, Ed.), Academic Press, New York, 1976.

[12] J. B. HTrIART-URRUTY, Conditions nécessaires d'optimalité en programmation non différentiable, C. R. Acad. Sci. Paris, 283 (1976), pp. 843-845.

[13] J. B. Hiriat-UnRuty, Gradients généralisés de fonctions composées. Applications, C. R. Acad. Sci. Paris, 285 (1977), pp. 781-874.

[14] J. B. HIRIART-URRUTY, Contributions à la programmation mathématique: cas déterministe et stochastique, Thèse, Université de Clermont II, 1977.

[15] A. D. Ioffe - L. Levin, Subbifferentials of convex functions, Trans. Moscow. Math. Soc., 26 (1972), pp. 1-72.

[16] G. J JMeson, Ordered Linear Spaces, Lectures Notes in Mathematics 141, Springer-Verlag, Berlin, 1970.

[17] I. KAWAI, Locally convex lattices, J. Math. Soc. Japan, 9 (1957), pp. 281-314.

[18] J. L. KExLer, General topology, Graduate texts in Mathematies, Springer-Verlag, New York, 1975.

[19] S. S. KutatididazE, Subdifferentials of convex operators, J. Math. Sibirski, 13 (1977), pp. 1057-1064 (in russian).

[20] G. LeBouRg, Valeur moyenne pour gradient généralisé, C. R. Acad. Sc. Paris, 281 (1975), pp. 795-797.

[21] C. Malivert - J. P. Penot - M. Thera, Un prolongement du théorème de Hahn-Banach, C. R. Acad. Sci. Paris, 286 (1978), pp. 165-168.

[22] P. MicheL, Problèmes des inégalités. Applications à la programmation et au contrôle optimal, Bull. Soc. Math. France, 101 (1973), pp. 413-439.

[23] P. MIchen, Problèmes des inégalités. Application à la programmation différentielle dans les espaces de Banach, C. R. Acad. Sci. Paris, 275 (1972), pp. 345-348.

[24] P. Micher, Problème d'optimisation défini par des fonotions qui sont sommes de fonctions convexes et de fonctions dérivables, J. Math. Pures et Appl., 53 (1974), pp. 321-330.

[25] R. MrFfLIN, Semismooth and semi-convex functions in constrained optimization, SIAM. J. Control, 15 (1977), pp. 959-972.

[26] J. J. Moreau, Fonetionnelles convexes, Séminaire sur les équations aux dérivées partielles, Collège de France, Paris, 1966-1967.

[27] N. Neumann, On the Strassen desintegration theorem, Arch. Math., 29 (1977), pp. 413-420.

[28] R. Pallo DF LA BarRIÈRe, Fonctions sous-linéarisables et principe de Pontryagin, Colloque sur le contrôle optimal, Bordeaux, 1973, Publications Mathématiques de l'Université de Bordeaux I, 1973-1974, (3), pp. 65-72.

13 - Annali di Matematica 
[29] J. P. Penot, Sous-différentiels de fonctions numériques non convexes, C. R. Acad. Sci. Paris, 278 (1974), pp. 1553-1555.

[30] J. P. Penot, Calcul sous-différentiel et optimisation, J. Functional Analysis, 27 (1978), pp. 248-276.

[31] J. P. Penot, Extrémisation et Optimisation, Cours d'analyse fonctionnelle appliquée, Pau, 1973-1974, multigraphié.

[32] A. L. Peressini, Ordered topological vector spaces, Harper's series in Modern Mathematics, Harper and Row, New York, 1967.

[33] B. H. Pourciat, Analysis and optimization of Lipschitz continous mappings, J. Optimization theory Appl., 22 (1977); pp. 311-351.

[34] C. RAFTr, Sur les programmes convexes définis dans des espaces vectoriels topologiques, Ann. Inst. Fourier, 20 (1970), pp. 457-491.

[35] R. T. Rockafellar, Oonvex Analysis, Princeton Math. Series no. 28, Princeton Univ. Press, Princeton, N. J., 1970.

[36] M. Robinson, Regularity and stability for convex multivalued functions, Math. of Operations Research, 1 (1976), pp, 130.143:

[37] J. SAINT-PIERRE, Une extension du théorème de Strassen, C. R. Acad. Sci. Paris, 279 (1974), pp. $5-8$.

[38] J. SAINT-PIERRE, Théorème de Strassen et intégration de sous-différentiels pour des fonctions à valeurs dans un espace vectoriel ordonné, Séminaire d'analyse convexe, Exposé no. 7, Montpellier, 1974.

[39] H. H. SchaEFFER, Topological vector spaces, Macmillan, New York, 1966.

[40] L. THIBAULt, Quelques propriétés des sous-différentiels de fonctions réelles localement lipschitziennes définies sur un espace de Banach séparable, C. R. Acad. Sci. Paris, 282 (1976), pp. 507-510.

[41] L. ThibautT, Quelques propriétés des sous-différentiels de fonctions localement lipschitziennes, Séminaire d'analyse convexe, Exposé no. 16, Montpellier, 1975.

[42] L. Thibault, Problème de Bolza dans un espace de Banach séparable, C. R. Acad. Sci. Paris, 282 (1976), pp. 1303-1306.

[43] M. VALADIER, Sous-différentiabilité de fonctions convexes à valeurs dans un espace vectoriel ordonné, Math. Scand., 30 (1972), pp. 65-72.

[44] J. WARGA, Derivate containers, inverse functions and controllability, in "Proceedings of MRC Symposium on the calculus of variations and optimal control, Sept. 1975 》(D. L. Russell, Ed.), Academic Press, New York, 1976.

[45] J. Zowe, Subdifferentiability of convex functions with values in an ordered vector space, Math. Scand., 34 (1974), pp. 69-83.

[46] J. ZowE, A duality theorem for a convex programming problem in order complete vector lattices, J. Math. Anal. Appl., 50 (1975), pp. 273-287. 